Supporting Information

\title{
Implications of Indenyl Substitution for the Structural Chemistry of Rare-Earth-Metal (Half-) Sandwich Complexes and Performance in Living Isoprene Polymerization
}

Dominic Diether, Cäcilia Maichle-Mössmer, and Reiner Anwander*

Institut für Anorganische Chemie, Eberhard Karls Universität Tübingen, Auf der Morgenstelle 18, 72076 Tübingen, Germany.

Corresponding Author

* reiner.anwander@uni-tuebingen.de 


\section{Table of Contents}

Figure S1. ${ }^{1} \mathrm{H}$ NMR spectrum of (Ind)La(AlMe $)_{2}$ (1a) $\quad$ S4

Figure $\mathrm{S} 2 .{ }^{13} \mathrm{C}\left\{{ }^{1} \mathrm{H}\right\}$ NMR spectrum of (Ind)La(AlMe 4$)_{2}(\mathbf{1 a}) \quad \mathrm{S} 4$

Figure $\mathrm{S} 3 .{ }^{1} \mathrm{H}^{13} \mathrm{C}-\mathrm{HSQC}$ NMR spectrum of (Ind)La(AlMe 4$)_{2}(\mathbf{1 a}) \quad$ S5

Figure $\mathrm{S} 4 .{ }^{1} \mathrm{H}^{1} \mathrm{H}-\mathrm{COSY}$ NMR spectrum of (Ind)La(AlMe $\left.)_{2}\right)_{2}(\mathbf{1 a}) \quad$ S5

Figure S5. ${ }^{1} \mathrm{H}$ NMR spectrum of $\left(\mathrm{Ind}^{\mathrm{Et}}\right) \mathrm{La}\left(\mathrm{AlMe}_{4}\right)_{2}(\mathbf{1 b}) \quad$ S6

Figure S6. ${ }^{13} \mathrm{C}\left\{{ }^{1} \mathrm{H}\right\}$ NMR spectrum of $\left(\mathrm{Ind}^{\mathrm{Et}}\right) \mathrm{La}\left(\mathrm{AlMe}_{4}\right)_{2}(\mathbf{1 b}) \quad$ S6

Figure S7. ${ }^{1} \mathrm{H}^{13} \mathrm{C}-\mathrm{HSQC}$ NMR spectrum of $\left(\mathrm{Ind}^{\mathrm{Et}}\right) \mathrm{La}\left(\mathrm{AlMe}_{4}\right)_{2}(\mathbf{1 b}) \quad$ S7

Figure S8. ${ }^{1} \mathrm{H}^{1} \mathrm{H}-\mathrm{COSY}$ NMR spectrum of $\left(\mathrm{Ind}^{\mathrm{Et}}\right) \mathrm{La}\left(\mathrm{AlMe}_{4}\right)_{2}(\mathbf{1 b}) \quad$ S8

Figure S9. ${ }^{1} \mathrm{H}$ NMR spectrum of $\left(\mathrm{Ind}^{\mathrm{tBu}}\right) \mathrm{La}\left(\mathrm{AlMe}_{4}\right)_{2}(\mathbf{1 c}) \quad$ S9

Figure $\mathrm{S} 10 .{ }^{13} \mathrm{C}\left\{{ }^{1} \mathrm{H}\right\}$ NMR spectrum of $\left(\mathrm{Ind}^{\mathrm{tBu}}\right) \mathrm{La}(\mathrm{AlMe})_{2}(\mathbf{1 c})$

Figure S11. ${ }^{1} \mathrm{H}^{13} \mathrm{C}-\mathrm{HSQC}$ NMR spectrum of $\left(\mathrm{Ind}^{\mathrm{tBu}}\right) \mathrm{La}\left(\mathrm{AlMe}_{4}\right)_{2}(\mathbf{1 c}) \quad \mathrm{S} 10$

Figure S12. ${ }^{1} \mathrm{H}^{1} \mathrm{H}-\mathrm{COSY}$ NMR spectrum of $\left(\mathrm{Ind}^{\mathrm{tBu}}\right) \mathrm{La}\left(\mathrm{AlMe}_{4}\right)_{2}(\mathbf{1 c}) \quad \mathrm{S} 11$

Figure S13. ${ }^{1} \mathrm{H}$ NMR spectrum of $\left(\mathrm{Ind}^{\mathrm{Si}}\right) \mathrm{La}\left(\mathrm{AlMe}_{4}\right)_{2}$ (1d) $\quad$ S12

Figure S14. ${ }^{13} \mathrm{C}\left\{{ }^{1} \mathrm{H}\right\}$ NMR spectrum of $\left(\mathrm{Ind}^{\mathrm{Si}}\right) \mathrm{La}\left(\mathrm{AlMe}_{4}\right)_{2}(\mathbf{1 d}) \quad \mathrm{S} 12$

Figure $\mathrm{S} 15 .{ }^{1} \mathrm{H}^{13} \mathrm{C}-\mathrm{HSQC}$ NMR spectrum of $\left(\mathrm{Ind}^{\mathrm{Si}}\right) \mathrm{La}\left(\mathrm{AlMe}_{4}\right)_{2}(\mathbf{1 d}) \quad \mathrm{S} 13$

Figure S16. ${ }^{1} \mathrm{H}^{1} \mathrm{H}-\mathrm{COSY}$ NMR spectrum of $\left(\mathrm{Ind}^{\mathrm{Si}}\right) \mathrm{La}\left(\mathrm{AlMe}_{4}\right)_{2}$ (1d) $\quad \mathrm{S} 14$

Figure S17. ${ }^{1} \mathrm{H}$ NMR spectrum of (Ind) $)_{2} \mathrm{Lu}\left(\mathrm{AlMe}_{4}\right)(\mathbf{2 a}) \quad \mathrm{S} 15$

Figure S18. ${ }^{13} \mathrm{C}\left\{{ }^{1} \mathrm{H}\right\}$ NMR spectrum of (Ind) $)_{2} \mathrm{Lu}\left(\mathrm{AlMe}_{4}\right)$ (2a)

Figure S19. ${ }^{1} \mathrm{H}^{13} \mathrm{C}-\mathrm{HSQC}$ NMR spectrum of $(\mathrm{Ind})_{2} \mathrm{Lu}\left(\mathrm{AlMe}_{4}\right)(\mathbf{2 a}) \quad \mathrm{S} 16$

Figure S20. ${ }^{1} \mathrm{H}^{1} \mathrm{H}$-COSY NMR spectrum of $(\mathrm{Ind})_{2} \mathrm{Lu}\left(\mathrm{AlMe}_{4}\right)$ (2a) $\quad \mathrm{S} 16$

Figure S21. ${ }^{1} \mathrm{H}$ NMR spectrum of $\left(\mathrm{Ind}^{\mathrm{Et}}\right)_{2} \mathrm{Lu}\left(\mathrm{AlMe}_{4}\right)(\mathbf{2 b}) \quad \mathrm{S} 17$

Figure $\mathrm{S} 22 .{ }^{13} \mathrm{C}\left\{{ }^{1} \mathrm{H}\right\}$ NMR spectrum of $\left(\mathrm{Ind}^{\mathrm{Et}}\right)_{2} \mathrm{Lu}\left(\mathrm{AlMe}_{4}\right)(\mathbf{2 b}) \quad \mathrm{S} 17$

Figure S23. ${ }^{1} \mathrm{H}^{13} \mathrm{C}-\mathrm{HSQC}$ NMR spectrum of $\left(\mathrm{Ind}^{\mathrm{Et}}\right) \mathrm{Lu}\left(\mathrm{AlMe}_{4}\right)(\mathbf{2 b}) \quad \mathrm{S} 18$

Figure S24. ${ }^{1} \mathrm{H}^{1} \mathrm{H}-\mathrm{COSY}$ NMR spectrum of $\left(\mathrm{Ind}^{\mathrm{Et}}\right) \mathrm{Lu}\left(\mathrm{AlMe}_{4}\right)(\mathbf{2 b}) \quad \mathrm{S} 19$

Figure S25. ${ }^{1} \mathrm{H}$ NMR spectrum of $\left(\mathrm{Ind}^{\mathrm{tBu}}\right)_{2} \mathrm{Lu}\left(\mathrm{AlMe}_{4}\right)(\mathbf{2 c}) \quad$ S20

Figure S26. ${ }^{13} \mathrm{C}\left\{{ }^{1} \mathrm{H}\right\}$ NMR spectrum of $\left(\mathrm{Ind}^{\mathrm{tBu}}\right)_{2} \mathrm{Lu}\left(\mathrm{AlMe}_{4}\right)(\mathbf{2 c}) \quad$ S20

Figure S27. ${ }^{1} \mathrm{H}^{13} \mathrm{C}-\mathrm{HSQC}$ NMR spectrum of $\left(\mathrm{Ind}^{t B u}\right)_{2} \mathrm{Lu}\left(\mathrm{AlMe}_{4}\right)(\mathbf{2 c}) \quad$ S21

Figure S28. ${ }^{1} \mathrm{H}^{1} \mathrm{H}-\mathrm{COSY}$ NMR spectrum of $\left(\mathrm{Ind}^{t B u}\right)_{2} \mathrm{Lu}\left(\mathrm{AlMe}_{4}\right)(\mathbf{2 c}) \quad$ S22

Figure S29. ${ }^{1} \mathrm{H}$ NMR spectrum of $\left(\mathrm{Ind}^{\mathrm{Si}}\right)_{2} \mathrm{Lu}\left(\mathrm{AlMe}_{4}\right)(\mathbf{2 d}) \quad$ S23

Figure S30. ${ }^{13} \mathrm{C}\left\{{ }^{1} \mathrm{H}\right\}$ NMR spectrum of $\left(\mathrm{Ind}^{\mathrm{Si}}\right)_{2} \mathrm{Lu}\left(\mathrm{AlMe}_{4}\right)(\mathbf{2 d}) \quad$ S23

Figure $\mathrm{S} 31 .{ }^{1} \mathrm{H}^{13} \mathrm{C}-\mathrm{HSQC}$ NMR spectrum of $\left(\mathrm{Ind}^{\mathrm{Si}}\right)_{2} \mathrm{Lu}\left(\mathrm{AlMe}_{4}\right)(\mathbf{2 d}) \quad$ S24

Figure S32. ${ }^{1} \mathrm{H}^{1} \mathrm{H}-\mathrm{COSY}$ NMR spectrum of $\left(\mathrm{Ind}^{\mathrm{Si}}\right)_{2} \mathrm{Lu}\left(\mathrm{AlMe}_{4}\right)(\mathbf{2 d}) \quad$ S25

Figure S33. ${ }^{29} \mathrm{Si}-{ }^{1} \mathrm{H}$ DEPT45 NMR spectrum of $\left(\mathrm{Ind}^{\mathrm{Si}}\right)_{2} \mathrm{Lu}\left(\mathrm{AlMe}_{4}\right)(\mathbf{2 d}) \quad$ S26

Figure $\mathrm{S} 34 .{ }^{1} \mathrm{H}^{29} \mathrm{Si}-\mathrm{HMBC}$ NMR spectrum of $\left(\mathrm{Ind}^{\mathrm{Si}}\right)_{2} \mathrm{Lu}\left(\mathrm{AlMe}_{4}\right)(\mathbf{2 d}) \quad \mathrm{S} 26$

Figure S35. VT ${ }^{1} \mathrm{H}$ NMR spectrum $\left(25-100^{\circ} \mathrm{C}\right)$ of $\left(\mathrm{Ind}^{\mathrm{Si}}\right)_{2} \mathrm{Lu}\left(\mathrm{AlMe}_{4}\right)(\mathbf{2 d}) \quad \mathrm{S} 27$

Figure S36. VT ${ }^{1} \mathrm{H}$ NMR spectrum $\left(-90-25^{\circ} \mathrm{C}\right)$ of $\left(\mathrm{Ind}^{\mathrm{Si}}\right)_{2} \mathrm{Lu}\left(\mathrm{AlMe}_{4}\right)(\mathbf{2 d}) \quad \mathrm{S} 28$

Figure S37. ${ }^{1} \mathrm{H}$ NMR spectrum of the reaction of (Ind) $\mathrm{La}\left(\mathrm{AlMe}_{4}\right)_{2}$ with $\left[\mathrm{Ph}_{3} \mathrm{C}\right]\left[\mathrm{B}\left(\mathrm{C}_{6} \mathrm{~F}_{5}\right)_{4}\right] \quad \mathrm{S} 29$

Figure S38. ${ }^{1} \mathrm{H}$ NMR spectrum of the reaction of (Ind) $\mathrm{La}\left(\mathrm{AlMe}_{4}\right)_{2}$ with $\left[\mathrm{PhNMe}_{2} \mathrm{H}\right]\left[\mathrm{B}\left(\mathrm{C}_{6} \mathrm{~F}_{5}\right)_{4}\right] \quad \mathrm{S} 29$

Figure S39. ${ }^{1} \mathrm{H}$ NMR spectrum of the reaction of (Ind) $\mathrm{La}\left(\mathrm{AlMe}_{4}\right)_{2}$ with $\mathrm{B}\left(\mathrm{C}_{6} \mathrm{~F}_{5}\right)_{3} \quad \mathrm{~S} 30$

Figure $\mathrm{S} 40 .{ }^{11} \mathrm{~B}$ NMR spectrum of the reaction of (Ind)La( $\left(\mathrm{AlMe}_{4}\right)_{2}$ with $\mathrm{B}\left(\mathrm{C}_{6} \mathrm{~F}_{5}\right)_{3} \quad \mathrm{~S} 30$

$\begin{array}{ll}\text { Figure S41. Molecular structure of }\left(\mathrm{Ind}^{\mathrm{Et}}\right) \mathrm{La}\left(\mathrm{AlMe}_{4}\right)_{2}(\mathbf{1 b}) & \mathrm{S} 31\end{array}$

Table S1. Crystallographic data for 1a, 1b, 1c, and 1d $\quad$ S32

Table S2. Crystallographic data for $\mathbf{2 a}, \mathbf{2 b}, \mathbf{2 c}$, and $\mathbf{2 d} \quad$ S32 
Figure S42. ${ }^{1} \mathrm{H}$ NMR spectrum of polyisoprene $\quad$ S34

Figure S43. ${ }^{13} \mathrm{C}\left\{{ }^{1} \mathrm{H}\right\}$ NMR spectrum of polyisoprene $\quad$ S34

Figure S44. GPC curve of polyisoprene $\quad$ S35

$\begin{array}{ll}\text { Figure S45. DSC curve of polyisoprene } & \text { S35 }\end{array}$

Figure S46. ${ }^{1} \mathrm{H}$ NMR spectrum of polyisoprene $\quad$ S36

Figure $\mathrm{S} 47 .{ }^{13} \mathrm{C}\left\{{ }^{1} \mathrm{H}\right\}$ NMR spectrum of polyisoprene $\quad$ S36

$\begin{array}{ll}\text { Figure S48. GPC curve of polyisoprene } & \text { S37 }\end{array}$

$\begin{array}{ll}\text { Figure S49. DSC curve of polyisoprene } & \text { S37 }\end{array}$ 


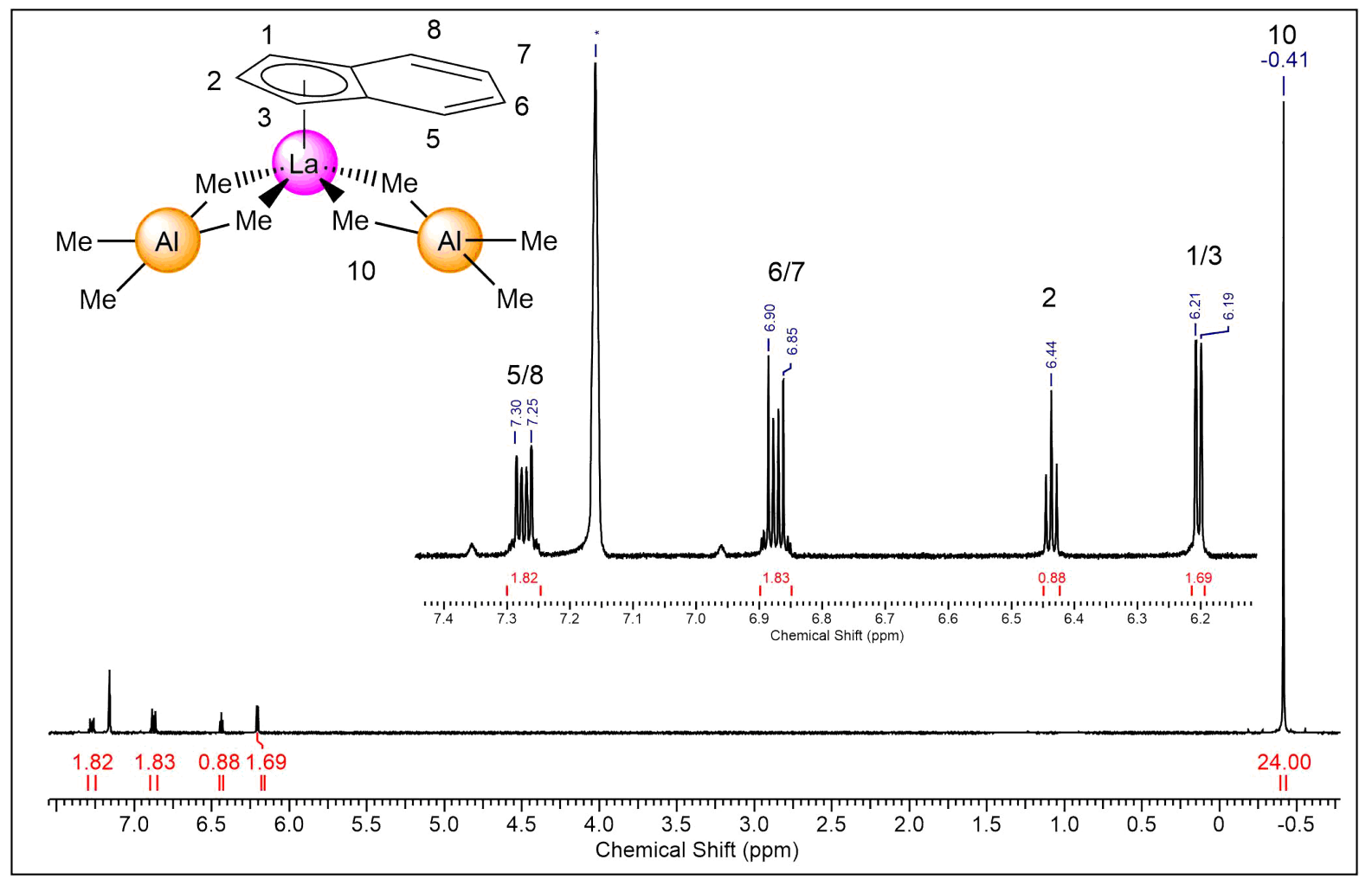

Figure S1. ${ }^{1} \mathrm{H}$ NMR spectrum $(400 \mathrm{MHz})$ of (Ind)La( $\left.\mathrm{AlMe}_{4}\right)_{2}(\mathbf{1 a})$ in $\mathrm{C}_{6} \mathrm{D}_{6}$ at $26{ }^{\circ} \mathrm{C}$.

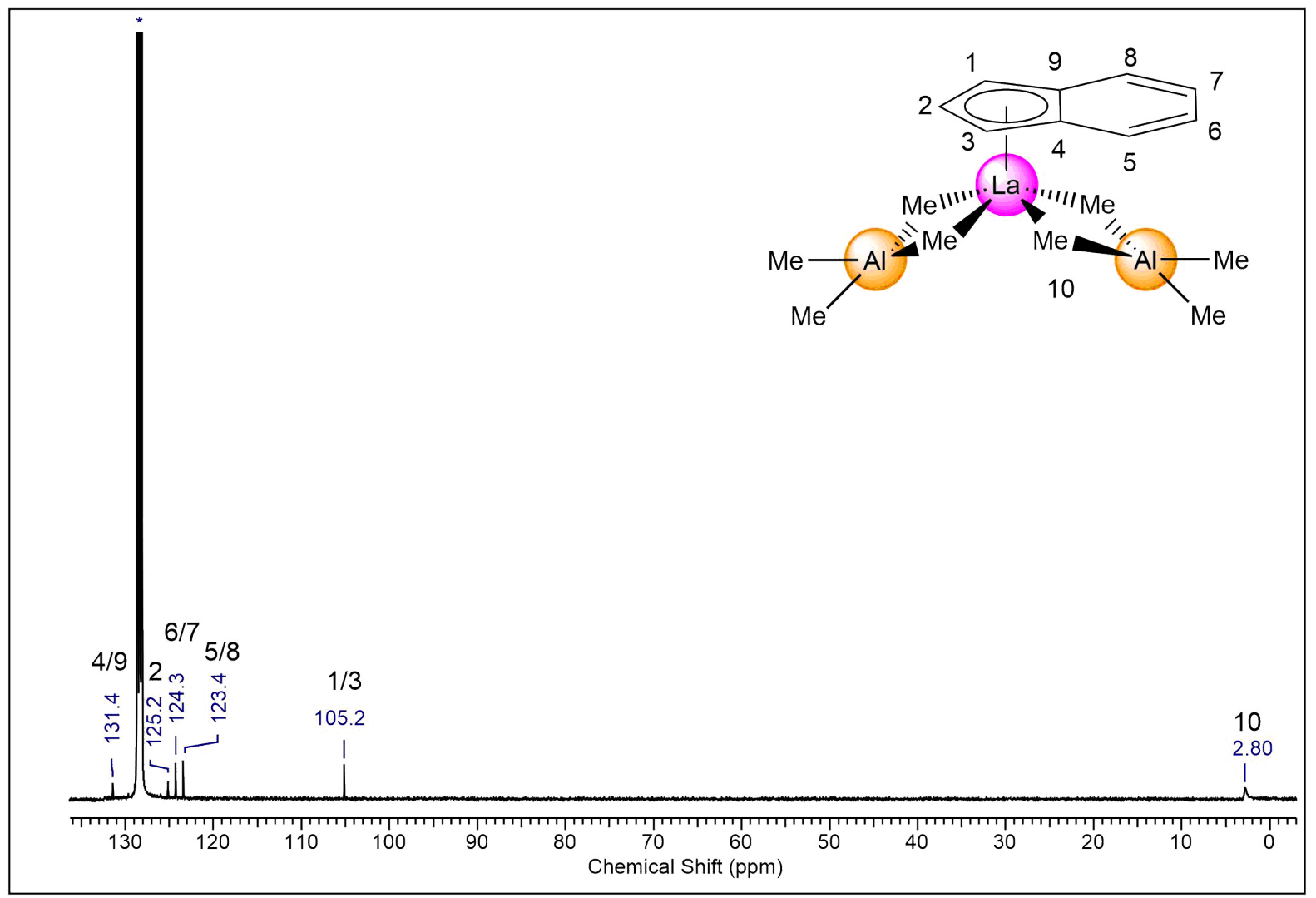

Figure $\mathbf{S 2} .{ }^{13} \mathrm{C}\left\{{ }^{1} \mathrm{H}\right\}$ NMR spectrum $(101 \mathrm{MHz})$ of (Ind) La(AlMe $)_{2}(\mathbf{1 a})$ in $\mathrm{C}_{6} \mathrm{D}_{6}$ at $26{ }^{\circ} \mathrm{C}$. 


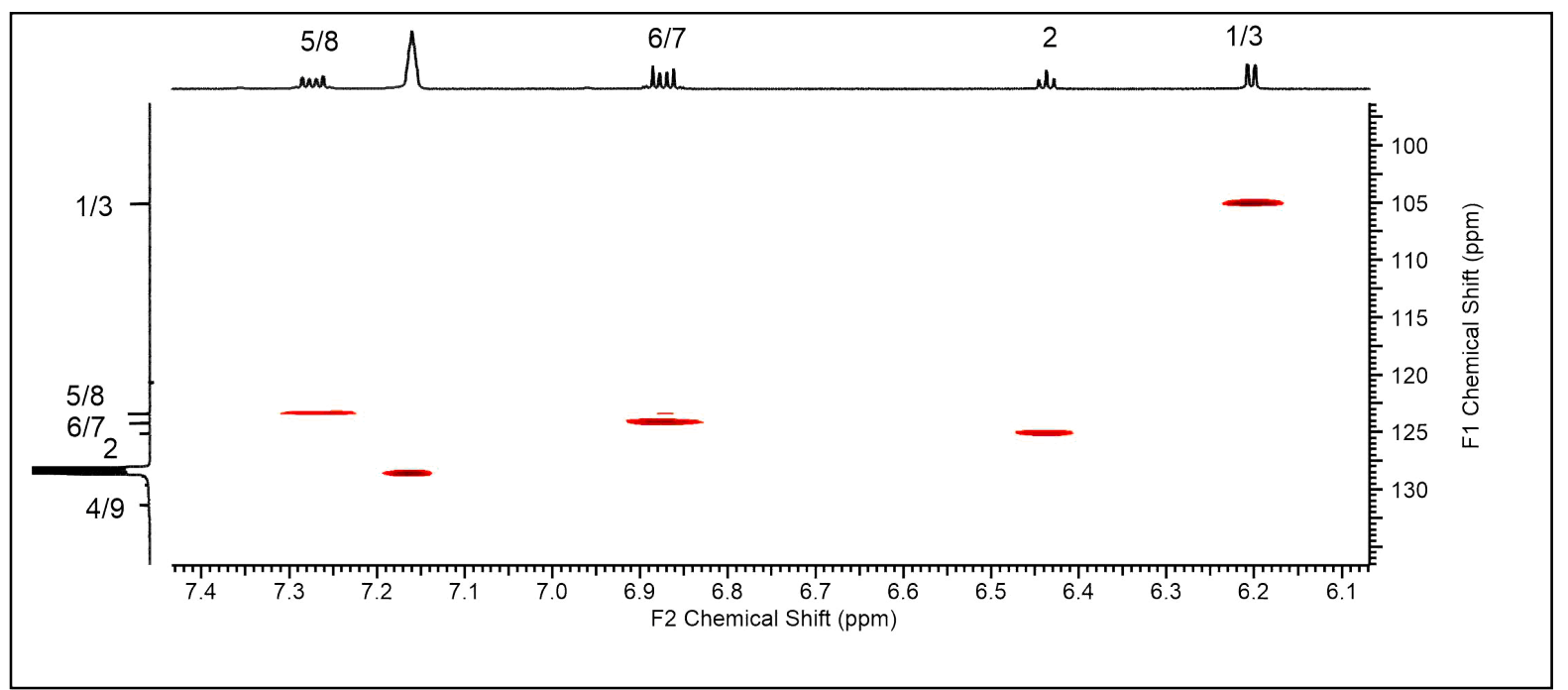

Figure S3. ${ }^{1} \mathrm{H}^{13} \mathrm{C}-\mathrm{HSQC}$ NMR spectrum $(400 / 101 \mathrm{MHz})$ of $(\mathrm{Ind}) \mathrm{La}\left(\mathrm{AlMe}_{4}\right)_{2}(\mathbf{1 a})$ in $\mathrm{C}_{6} \mathrm{D}_{6}$ at $26{ }^{\circ} \mathrm{C}$.

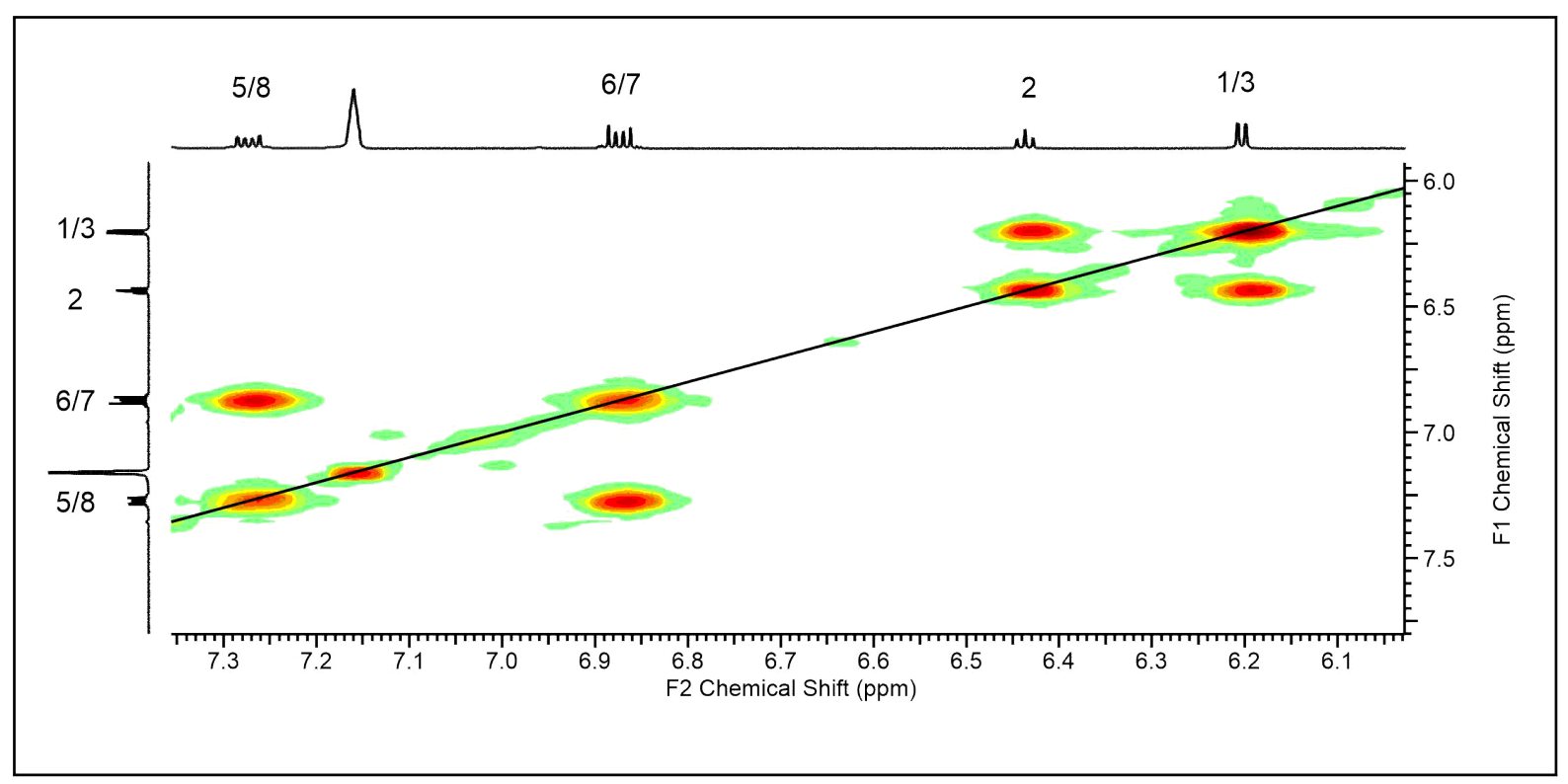

Figure S4. Section of the ${ }^{1} \mathrm{H}^{1} \mathrm{H}-\mathrm{COSY}$ NMR spectrum $(400 / 400 \mathrm{MHz})$ of (Ind)La(AlMe $)_{2}(\mathbf{1 a})$ in $\mathrm{C}_{6} \mathrm{D}_{6}$ at $26^{\circ} \mathrm{C}$. 




Figure S5. ${ }^{1} \mathrm{H}$ NMR spectrum $(400 \mathrm{MHz})$ of $\left(\mathrm{Ind}^{\mathrm{Et}}\right) \mathrm{La}\left(\mathrm{AlMe}_{4}\right)_{2}(\mathbf{1 b})$ in $\mathrm{C}_{6} \mathrm{D}_{6}$ at $26^{\circ} \mathrm{C}$.

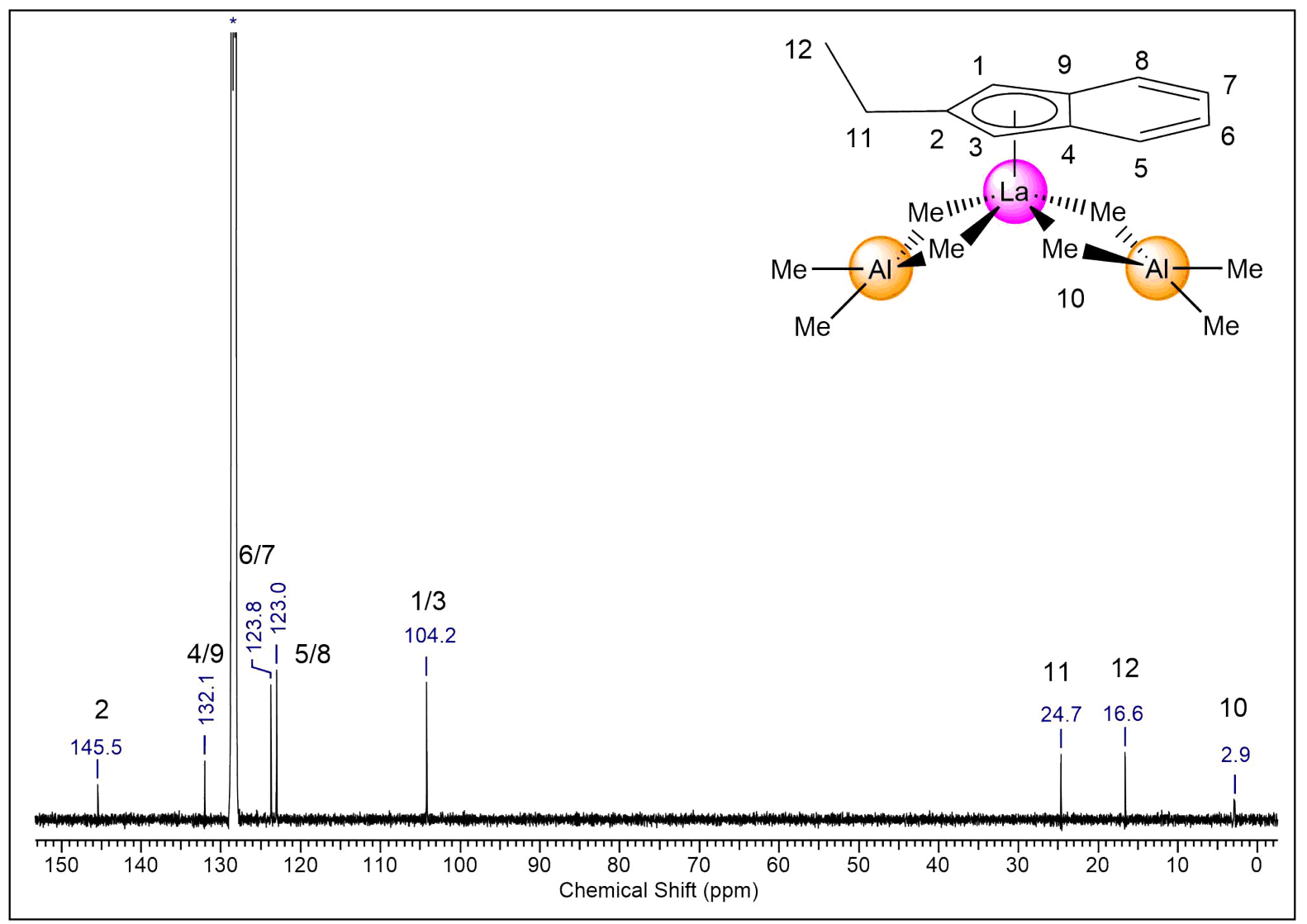

Figure S6. ${ }^{13} \mathrm{C}\left\{{ }^{1} \mathrm{H}\right\}$ NMR spectrum $(101 \mathrm{MHz})$ of $\left(\operatorname{Ind}^{\mathrm{Et}}\right) \mathrm{La}\left(\mathrm{AlMe}_{4}\right)_{2}(\mathbf{1 b})$ in $\mathrm{C}_{6} \mathrm{D}_{6}$ at $26{ }^{\circ} \mathrm{C}$. 

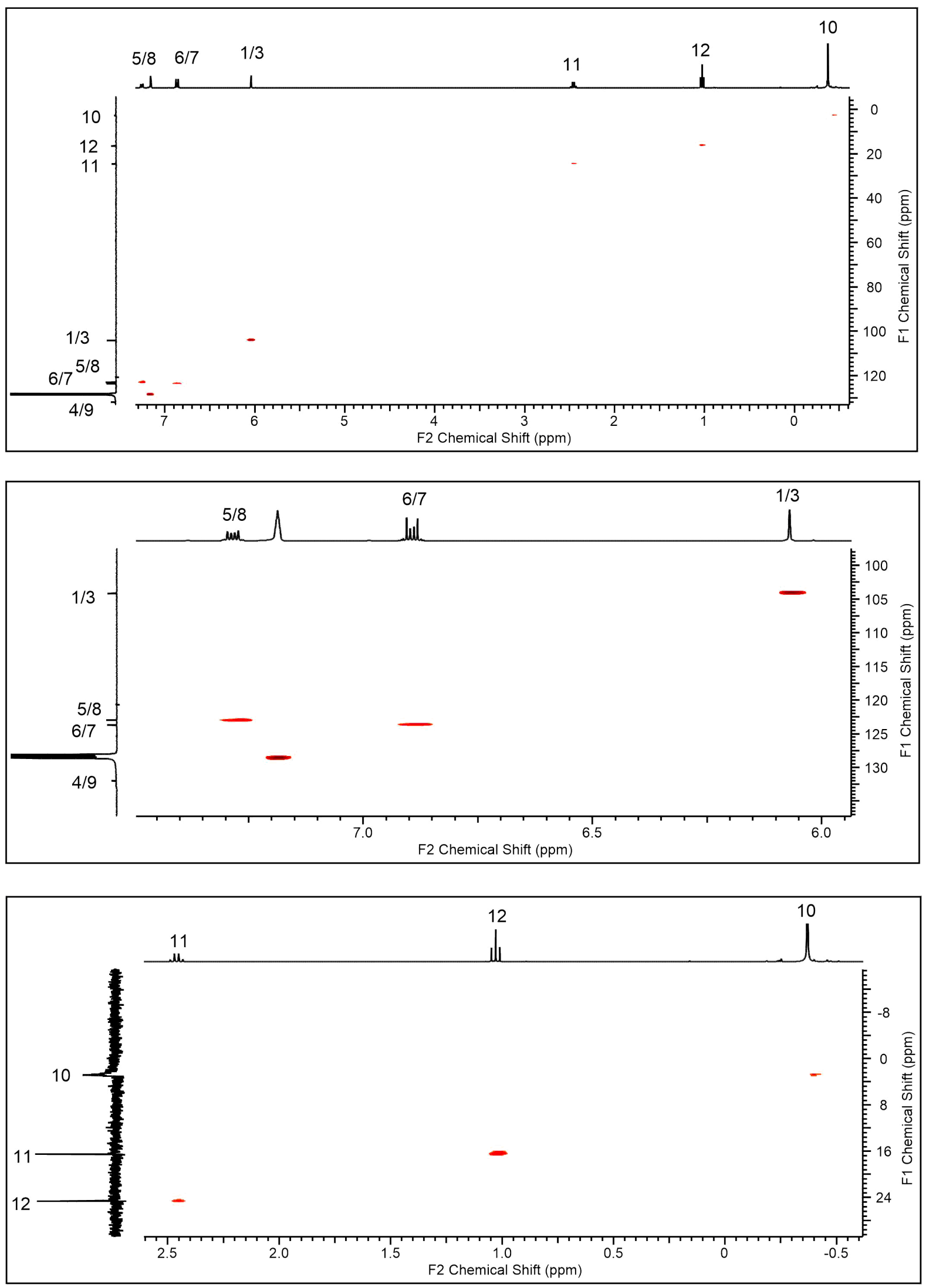

Figure S7. ${ }^{1} \mathrm{H}^{13} \mathrm{C}-\mathrm{HSQC}$ NMR spectrum $(400 / 101 \mathrm{MHz})$ of $\left(\mathrm{Ind}^{\mathrm{Et}}\right) \mathrm{La}\left(\mathrm{AlMe}_{4}\right)_{2}(\mathbf{1 b})$ in $\mathrm{C}_{6} \mathrm{D}_{6}$ at $26^{\circ} \mathrm{C}$, including enlarged sections. 

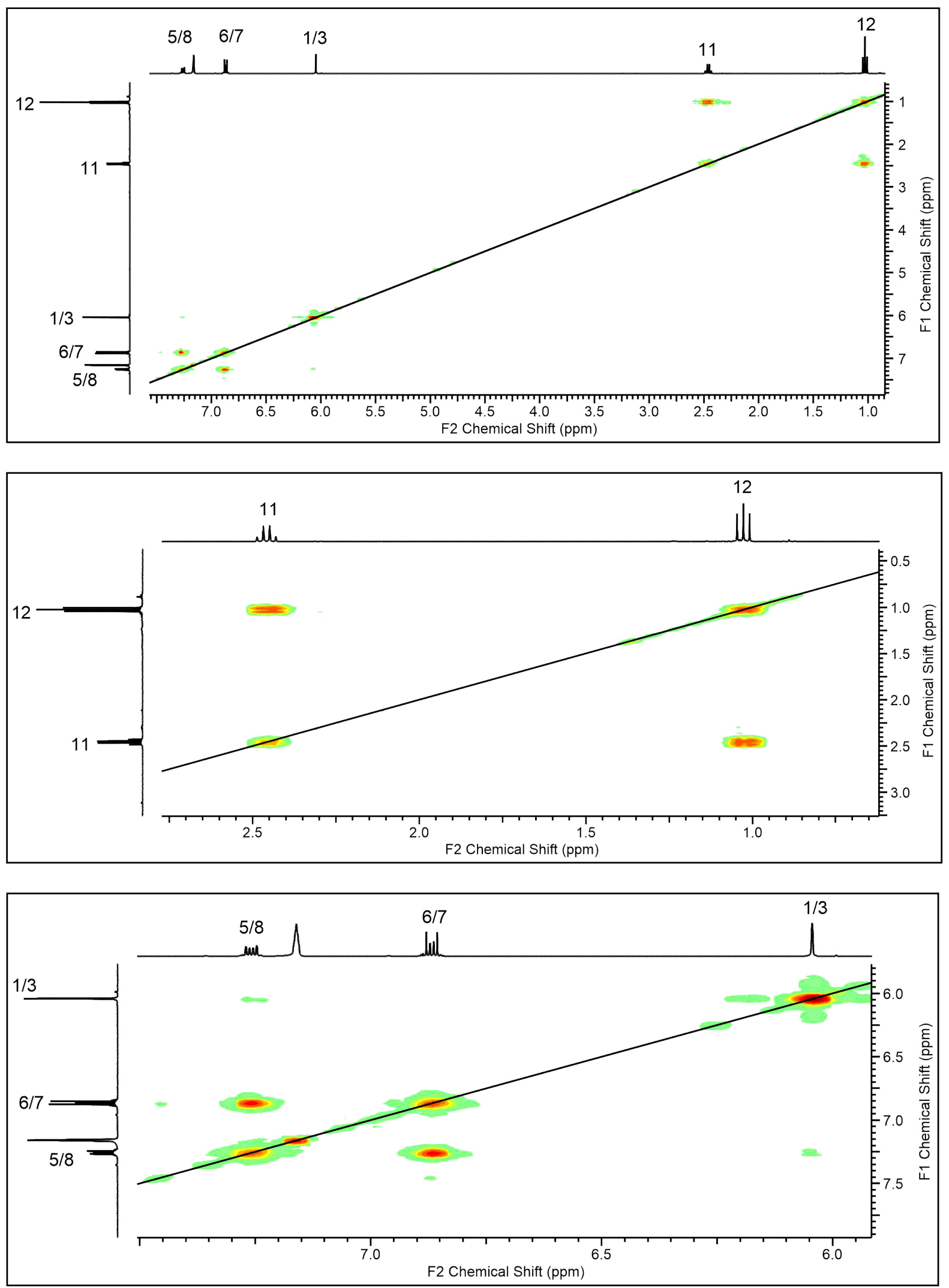

Figure S8. ${ }^{1} \mathrm{H}^{1} \mathrm{H}-\mathrm{COSY}$ NMR spectrum $(400 / 400 \mathrm{MHz})$ of $\left(\mathrm{Ind}^{\mathrm{Et}}\right) \mathrm{La}\left(\mathrm{AlMe}_{4}\right)_{2}(\mathbf{1 b})$ in $\mathrm{C}_{6} \mathrm{D}_{6}$ at $26{ }^{\circ} \mathrm{C}$, including enlarged sections. 


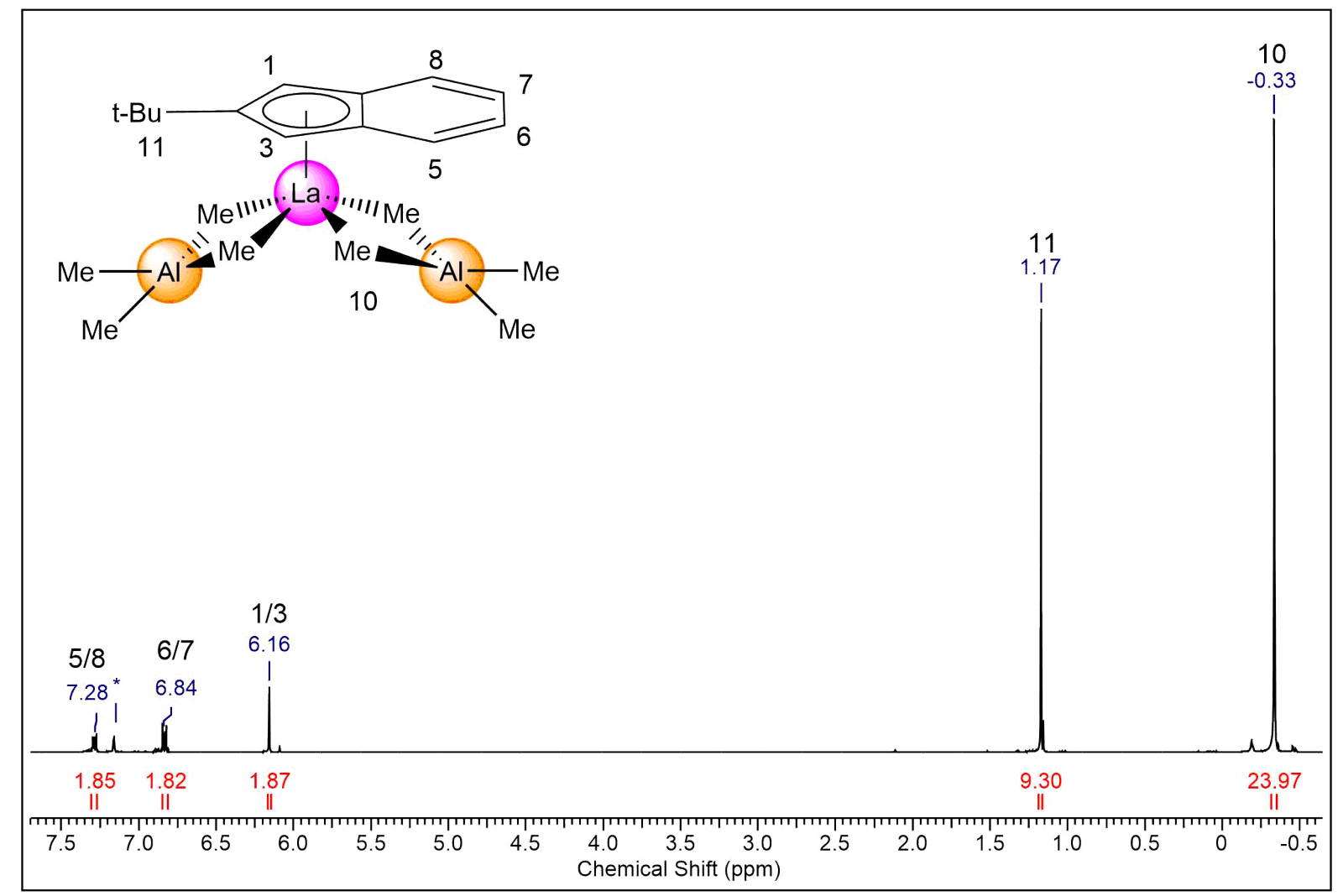

Figure S9. ${ }^{1} \mathrm{H}$ NMR spectrum $(400 \mathrm{MHz})$ of $\left(\mathrm{Ind}^{\mathrm{BBu}}\right) \mathrm{La}\left(\mathrm{AlMe}_{4}\right)_{2}(\mathbf{1 c})$ in $\mathrm{C}_{6} \mathrm{D}_{6}$ at $26{ }^{\circ} \mathrm{C}$.

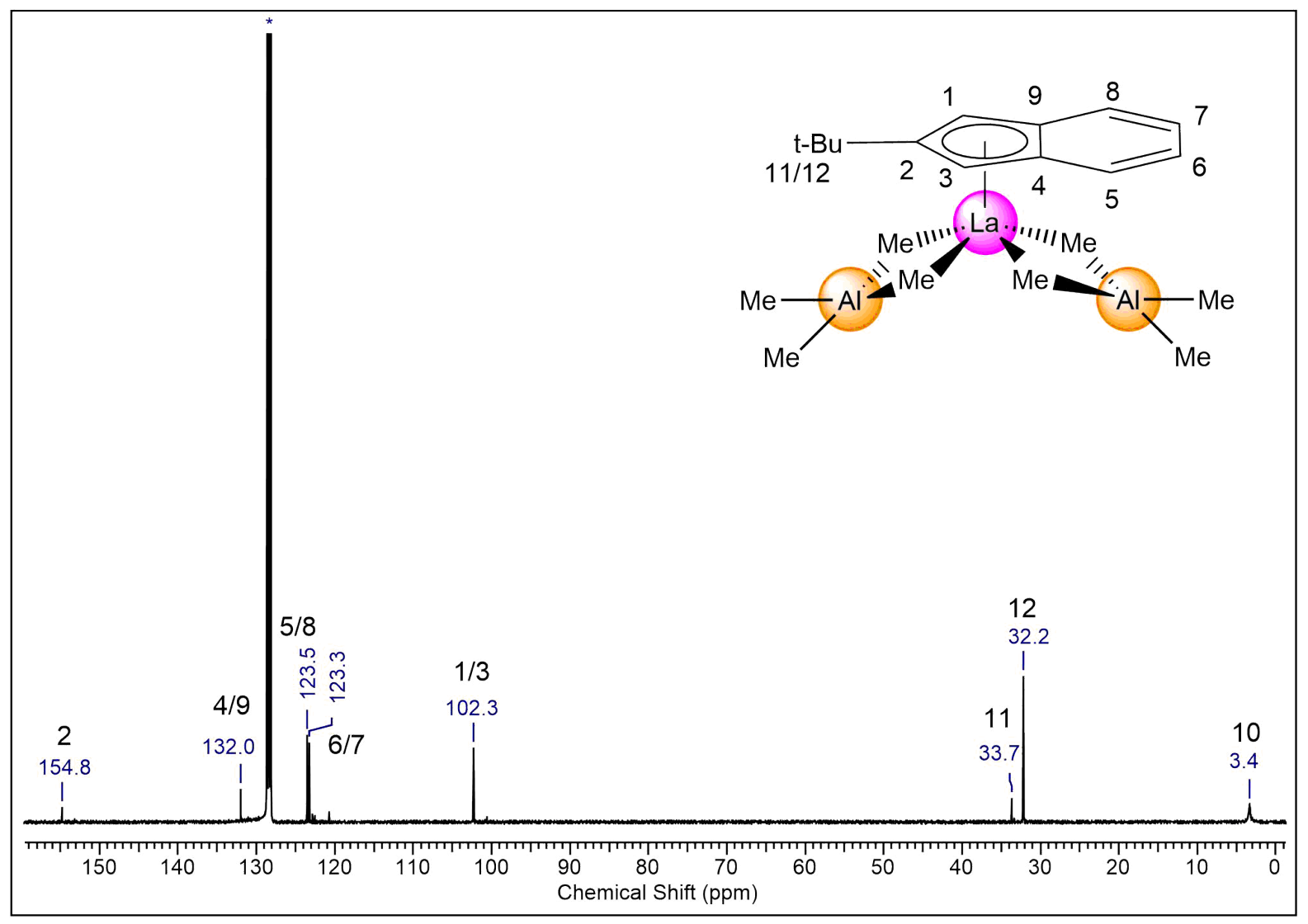

Figure S10. ${ }^{13} \mathrm{C}\left\{{ }^{1} \mathrm{H}\right\}$ NMR spectrum $(101 \mathrm{MHz})$ of $\left(\operatorname{Ind}^{t \mathrm{Bu}}\right) \mathrm{La}\left(\mathrm{AlMe}_{4}\right)_{2}(\mathbf{1 c})$ in $\mathrm{C}_{6} \mathrm{D}_{6}$ at $26{ }^{\circ} \mathrm{C}$. 

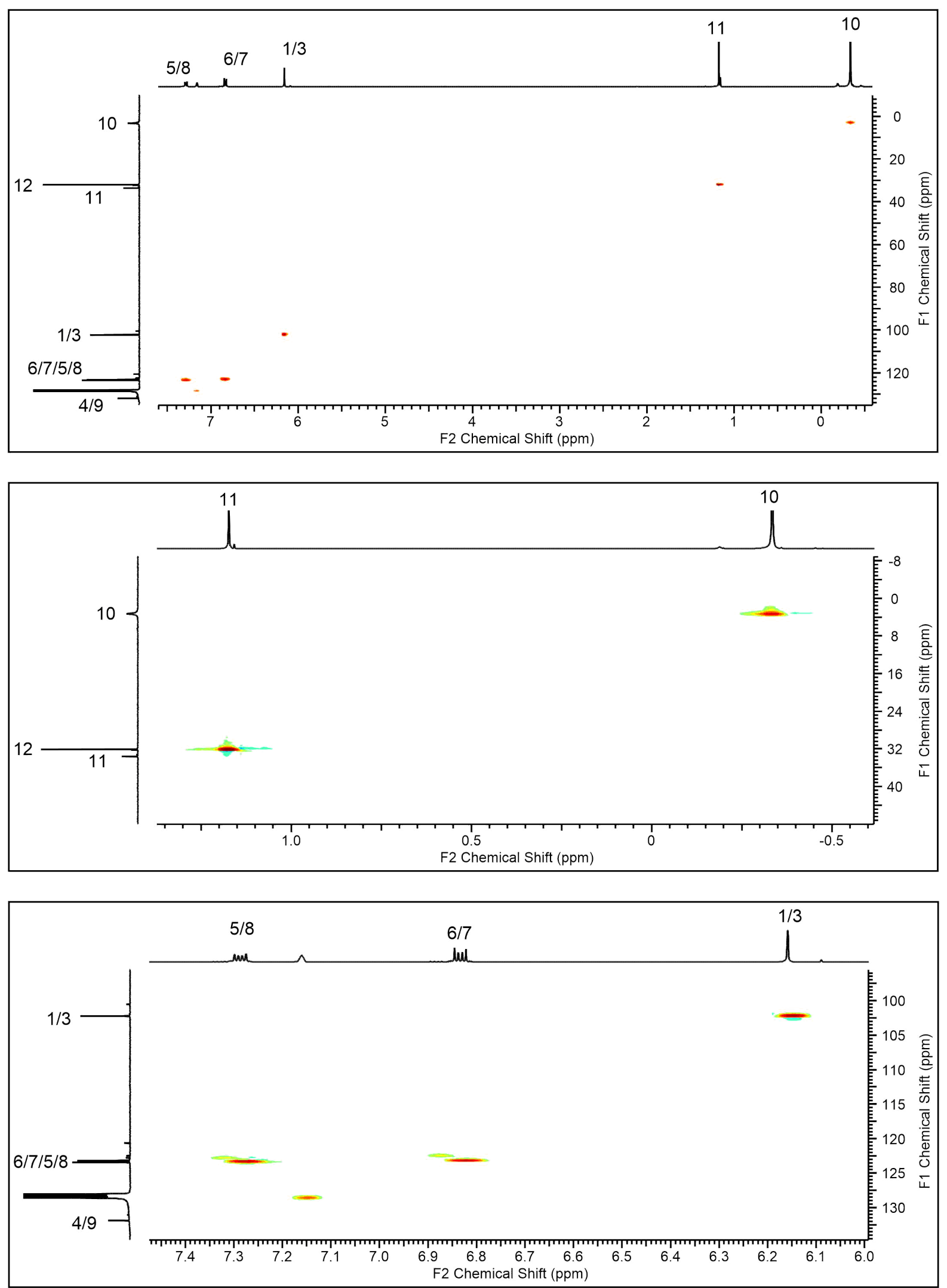

Figure S11. ${ }^{1} \mathrm{H}^{13} \mathrm{C}-\mathrm{HSQC}$ NMR spectrum $(400 / 101 \mathrm{MHz})$ of $\left(\operatorname{Ind}^{t B u}\right) \mathrm{La}\left(\mathrm{AlMe}_{4}\right)_{2}(\mathbf{1 c})$ in $\mathrm{C}_{6} \mathrm{D}_{6}$ at $26^{\circ} \mathrm{C}$, including enlarged sections. 

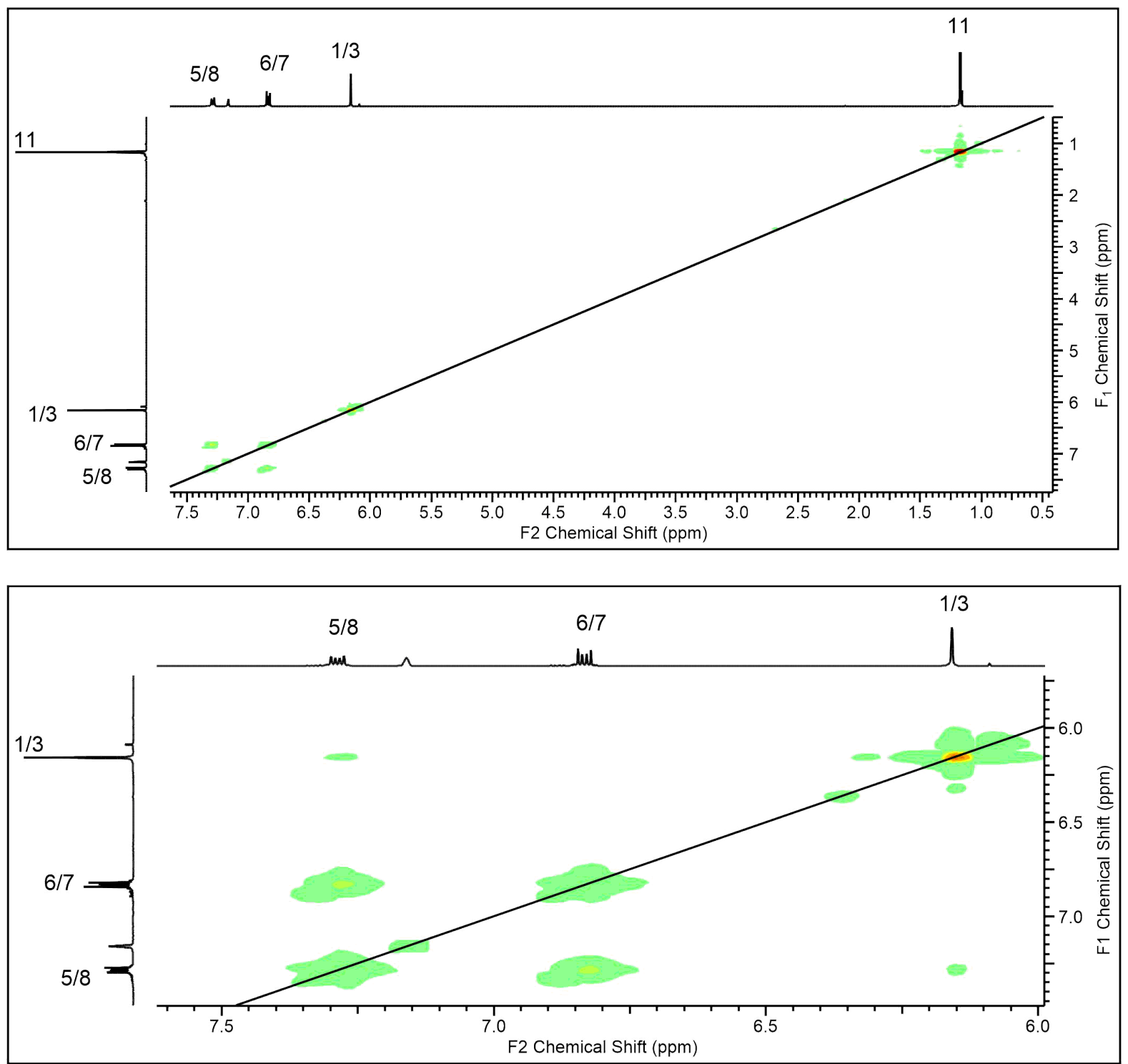

Figure S12. ${ }^{1} \mathrm{H}^{1} \mathrm{H}-\mathrm{COSY}$ NMR spectrum $(400 / 400 \mathrm{MHz})$ of $\left(\operatorname{Ind}^{t B u}\right) \mathrm{La}\left(\mathrm{AlMe}_{4}\right)_{2}(\mathbf{1 c})$ in $\mathrm{C}_{6} \mathrm{D}_{6}$ at $26^{\circ} \mathrm{C}$, including an enlarged section. 


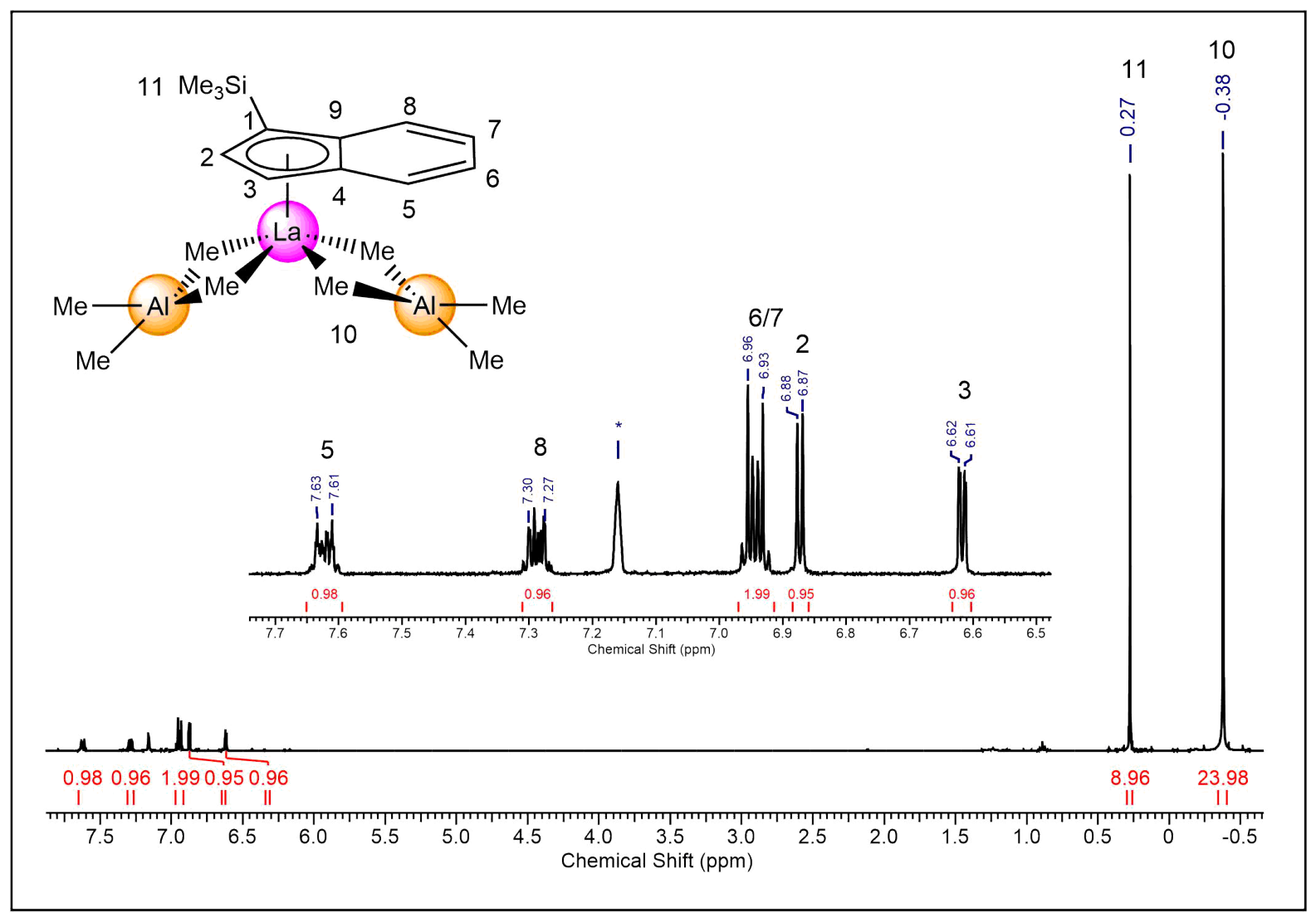

Figure S13. ${ }^{1} \mathrm{H}$ NMR spectrum $(400 \mathrm{MHz})$ of $\left(\mathrm{Ind}^{\mathrm{Si}}\right) \mathrm{La}\left(\mathrm{AlMe}_{4}\right)_{2}(\mathbf{1 d})$ in $\mathrm{C}_{6} \mathrm{D}_{6}$ at $26^{\circ} \mathrm{C}$.

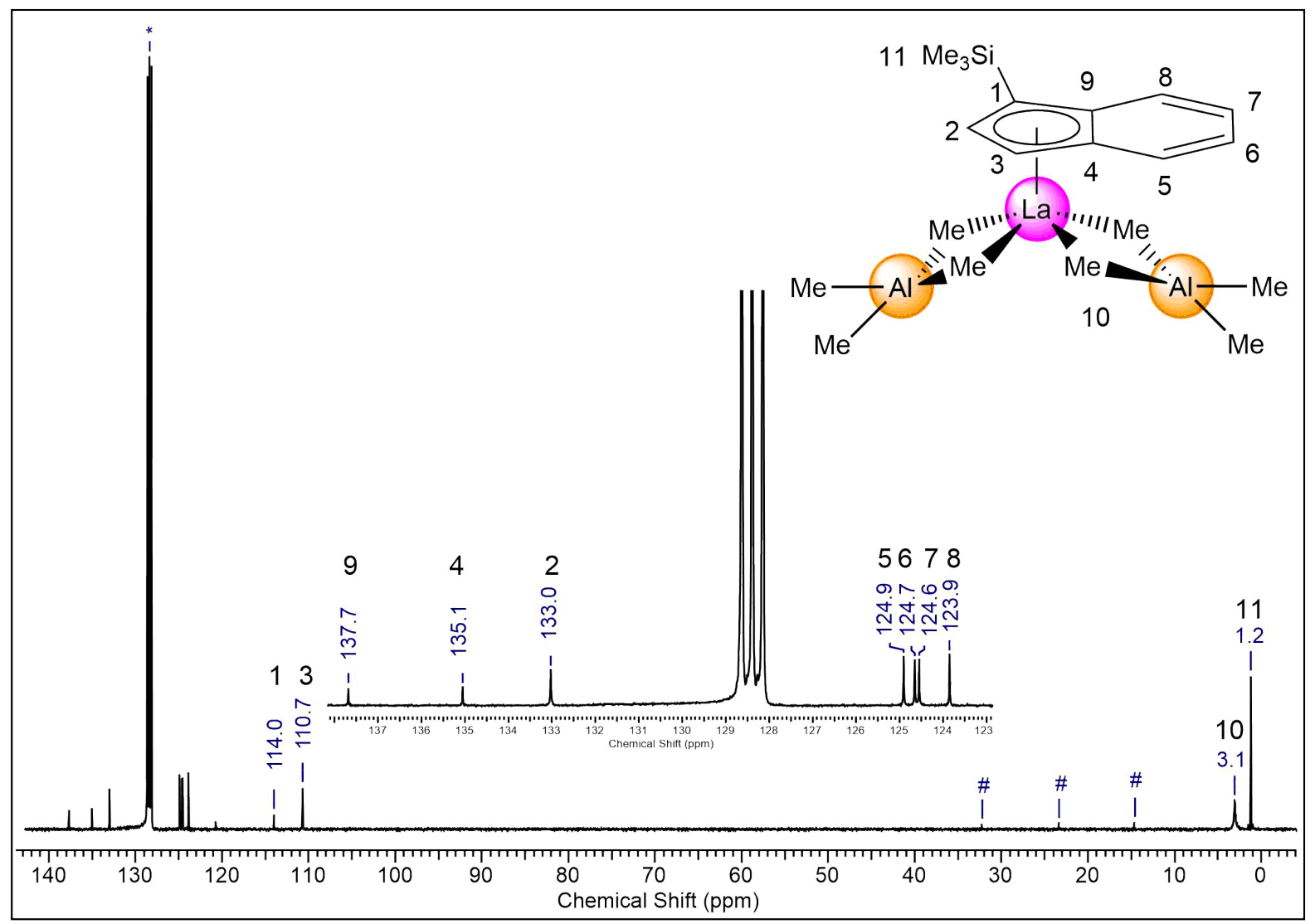

Figure S14. ${ }^{13} \mathrm{C}\left\{{ }^{1} \mathrm{H}\right\}$ NMR spectrum $(101 \mathrm{MHz})$ of $\left(\operatorname{Ind}^{\mathrm{Si}}\right) \mathrm{La}\left(\mathrm{AlMe}_{4}\right)_{2}(\mathbf{1 d})$ in $\mathrm{C}_{6} \mathrm{D}_{6}$ at $26{ }^{\circ} \mathrm{C}$. Residual $n$-hexane marked with \#. 

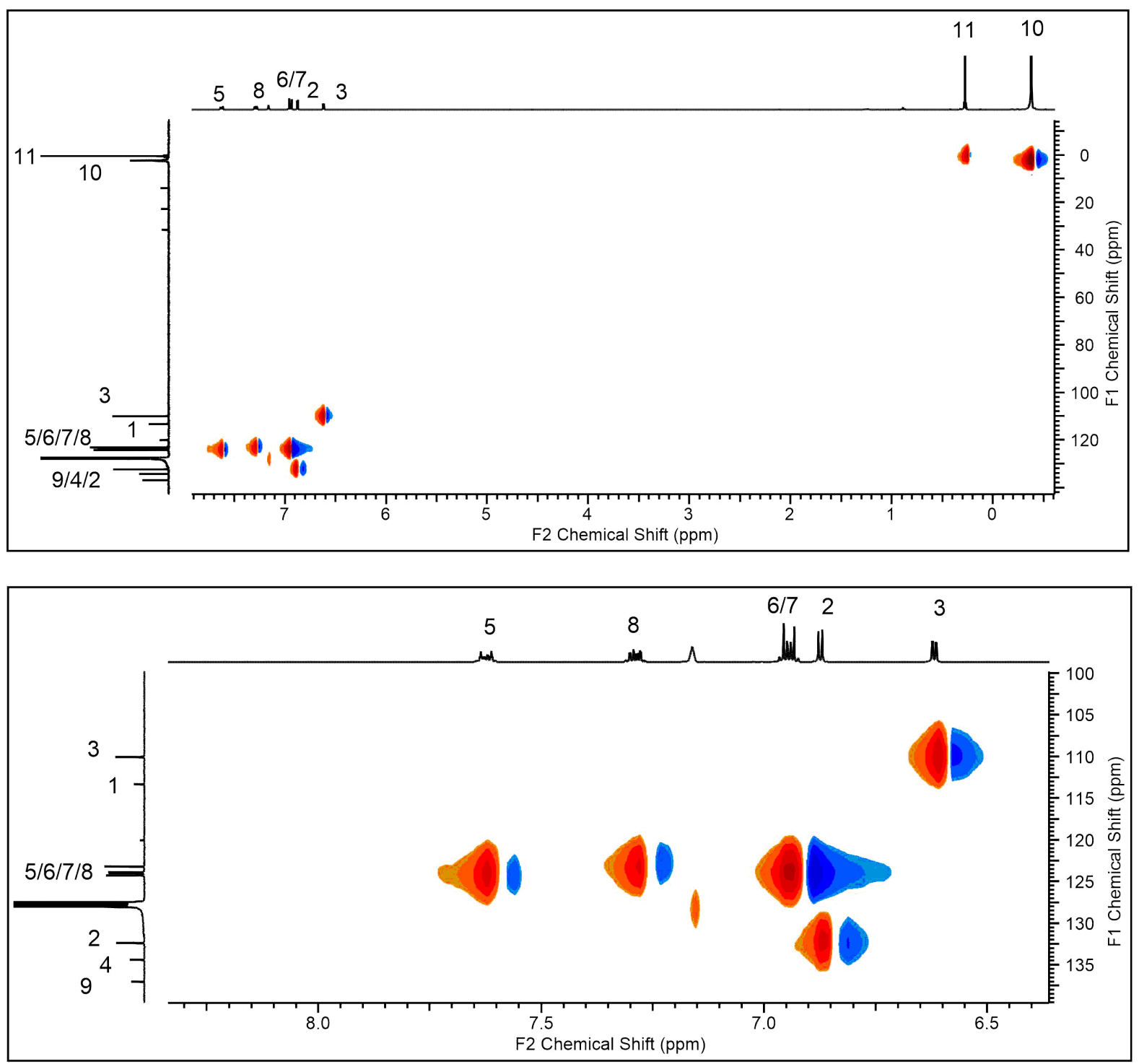

Figure S15. ${ }^{1} \mathrm{H}^{13} \mathrm{C}-\mathrm{HSQC}$ NMR spectrum $(400 / 101 \mathrm{MHz})$ of $\left(\mathrm{Ind}^{\mathrm{Si}}\right) \mathrm{La}\left(\mathrm{AlMe}_{4}\right)_{2}(\mathbf{1 d})$ in $\mathrm{C}_{6} \mathrm{D}_{6}$ at $26{ }^{\circ} \mathrm{C}$, including an enlarged section. 

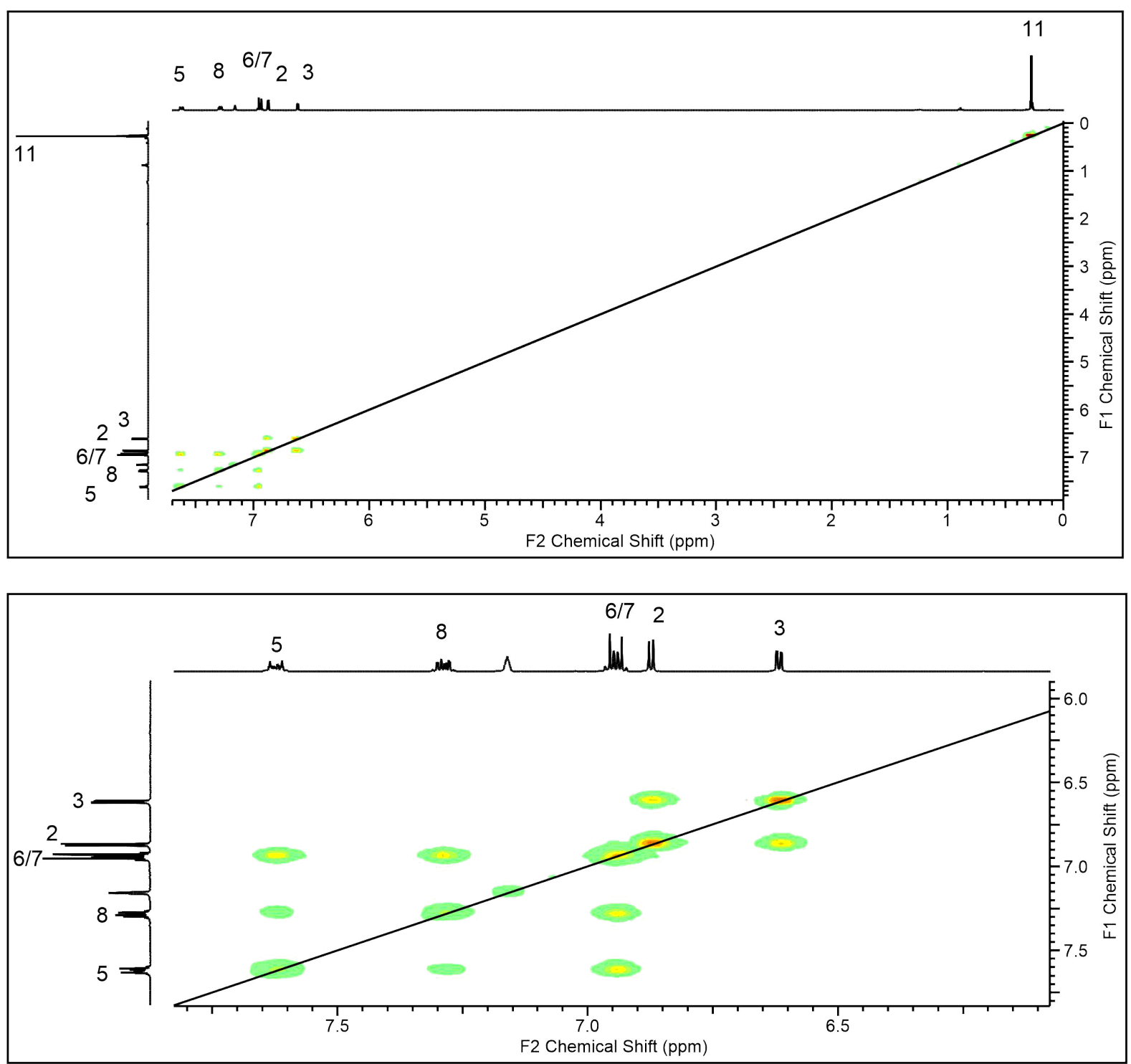

Figure S16. ${ }^{1} \mathrm{H}^{1} \mathrm{H}-\mathrm{COSY}$ NMR spectrum $(400 / 400 \mathrm{MHz})$ of $\left(\mathrm{Ind}^{\mathrm{Si}}\right) \mathrm{La}\left(\mathrm{AlMe}_{4}\right)_{2}(\mathbf{1 d})$ in $\mathrm{C}_{6} \mathrm{D}_{6}$ at $26{ }^{\circ} \mathrm{C}$, including an enlarged section. 


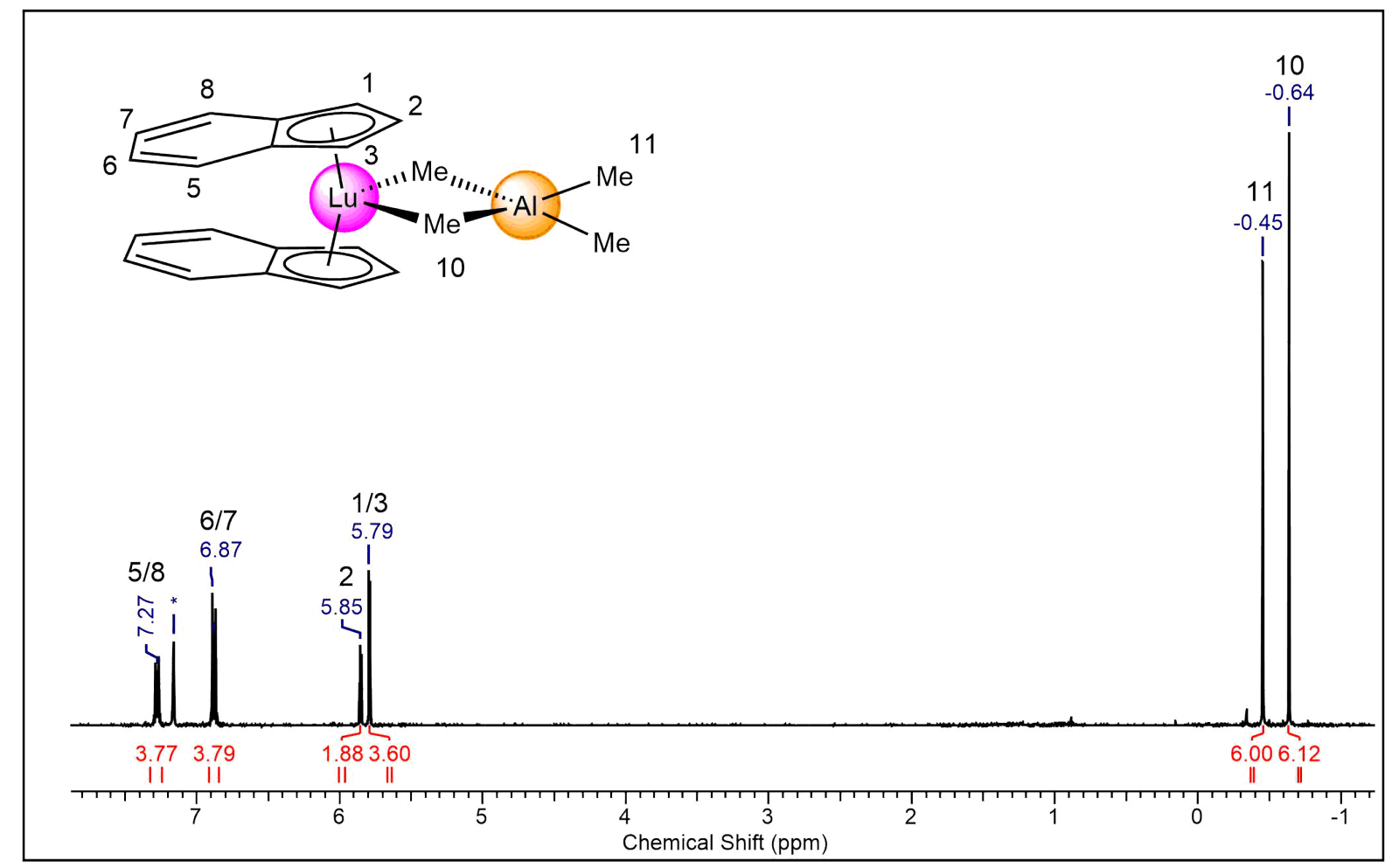

Figure S17. ${ }^{1} \mathrm{H}$ NMR spectrum $(400 \mathrm{MHz})$ of $(\mathrm{Ind})_{2} \mathrm{Lu}\left(\mathrm{AlMe}_{4}\right)(\mathbf{2 a})$ in $\mathrm{C}_{6} \mathrm{D}_{6}$ at $26{ }^{\circ} \mathrm{C}$.

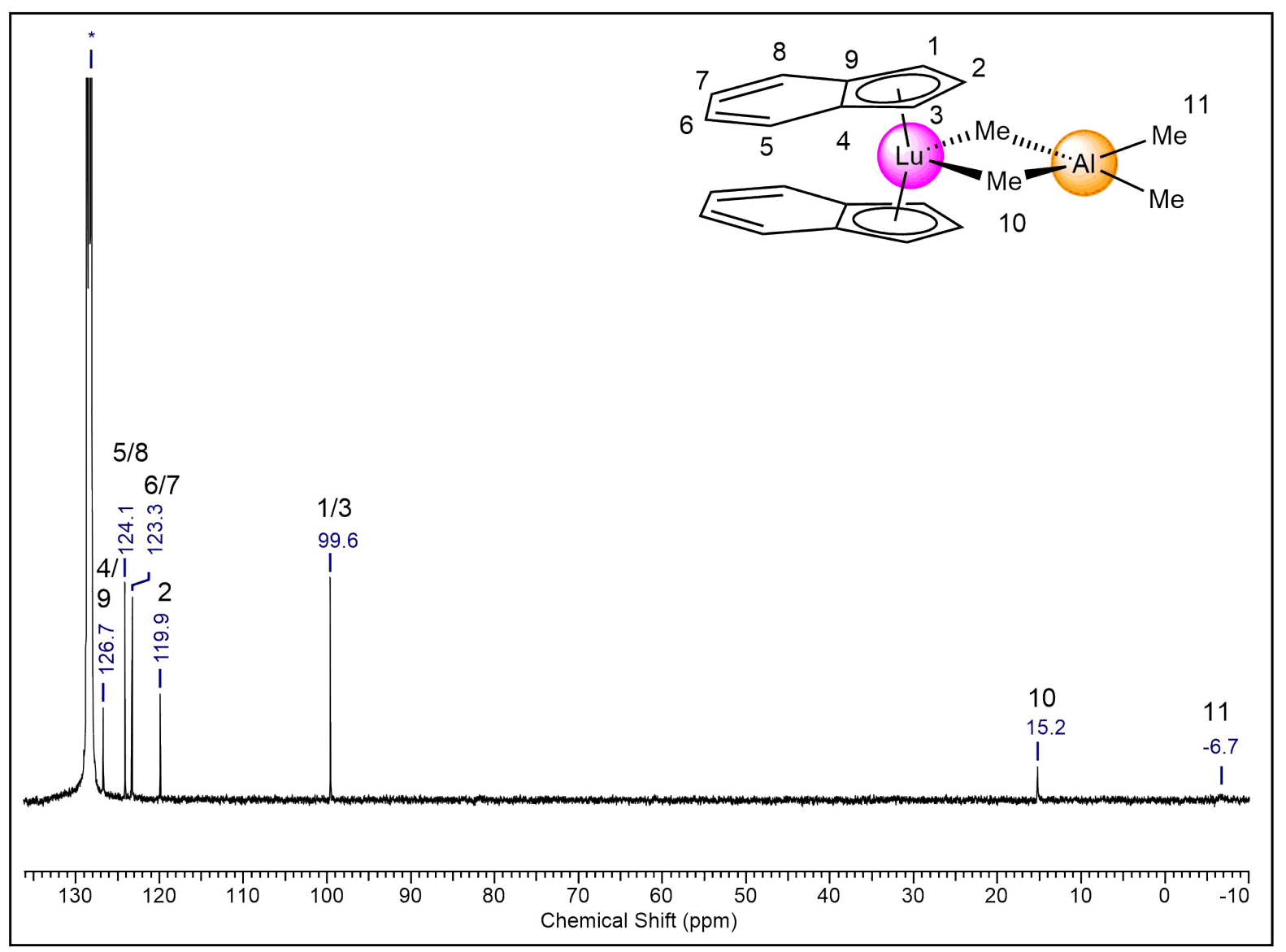

Figure S18. ${ }^{13} \mathrm{C}\left\{{ }^{1} \mathrm{H}\right\}$ NMR spectrum $(101 \mathrm{MHz})$ of $(\mathrm{Ind})_{2} \mathrm{Lu}\left(\mathrm{AlMe}_{4}\right)(\mathbf{2 a})$ in $\mathrm{C}_{6} \mathrm{D}_{6}$ at $26{ }^{\circ} \mathrm{C}$. 




Figure S19. Section of the ${ }^{1} \mathrm{H}^{13} \mathrm{C}-\mathrm{HSQC}$ NMR spectrum $(400 / 101 \mathrm{MHz})$ of $(\mathrm{Ind})_{2} \mathrm{Lu}\left(\mathrm{AlMe}_{4}\right)(\mathbf{2 a})$ in $\mathrm{C}_{6} \mathrm{D}_{6}$ at $26{ }^{\circ} \mathrm{C}$.

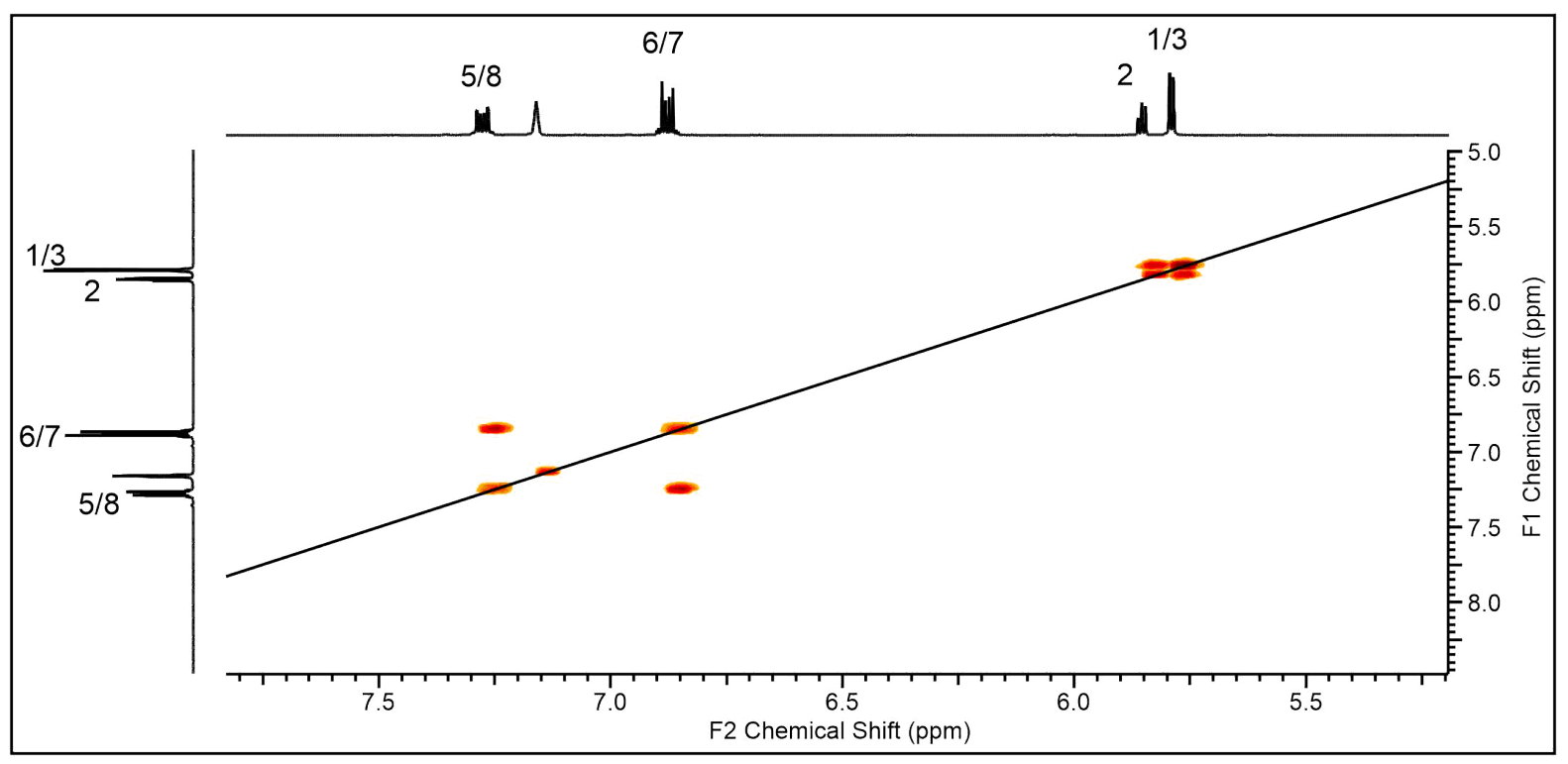

Figure S20. Section of the ${ }^{1} \mathrm{H}^{1} \mathrm{H}-\mathrm{COSY}$ NMR spectrum $(400 / 400 \mathrm{MHz})$ of (Ind) $)_{2} \mathrm{Lu}\left(\mathrm{AlMe}_{4}\right)(\mathbf{2 a})$ in $\mathrm{C}_{6} \mathrm{D}_{6}$ at $26^{\circ} \mathrm{C}$. 


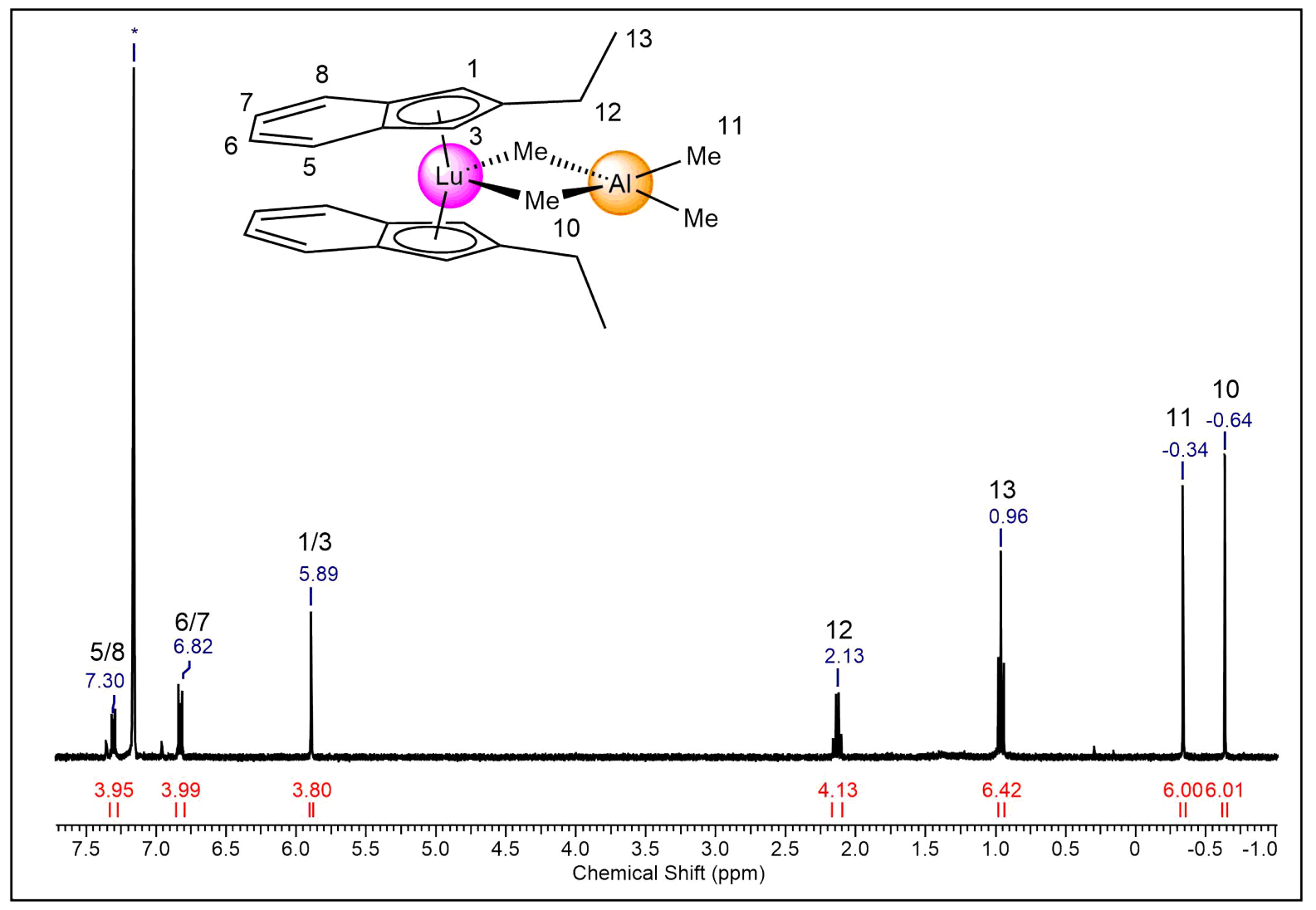

Figure S21. ${ }^{1} \mathrm{H}$ NMR spectrum $(400 \mathrm{MHz})$ of $\left(\mathrm{Ind}^{\mathrm{Et}}\right)_{2} \mathrm{Lu}\left(\mathrm{AlMe}_{4}\right)(\mathbf{2 b})$ in $\mathrm{C}_{6} \mathrm{D}_{6}$ at $26^{\circ} \mathrm{C}$.

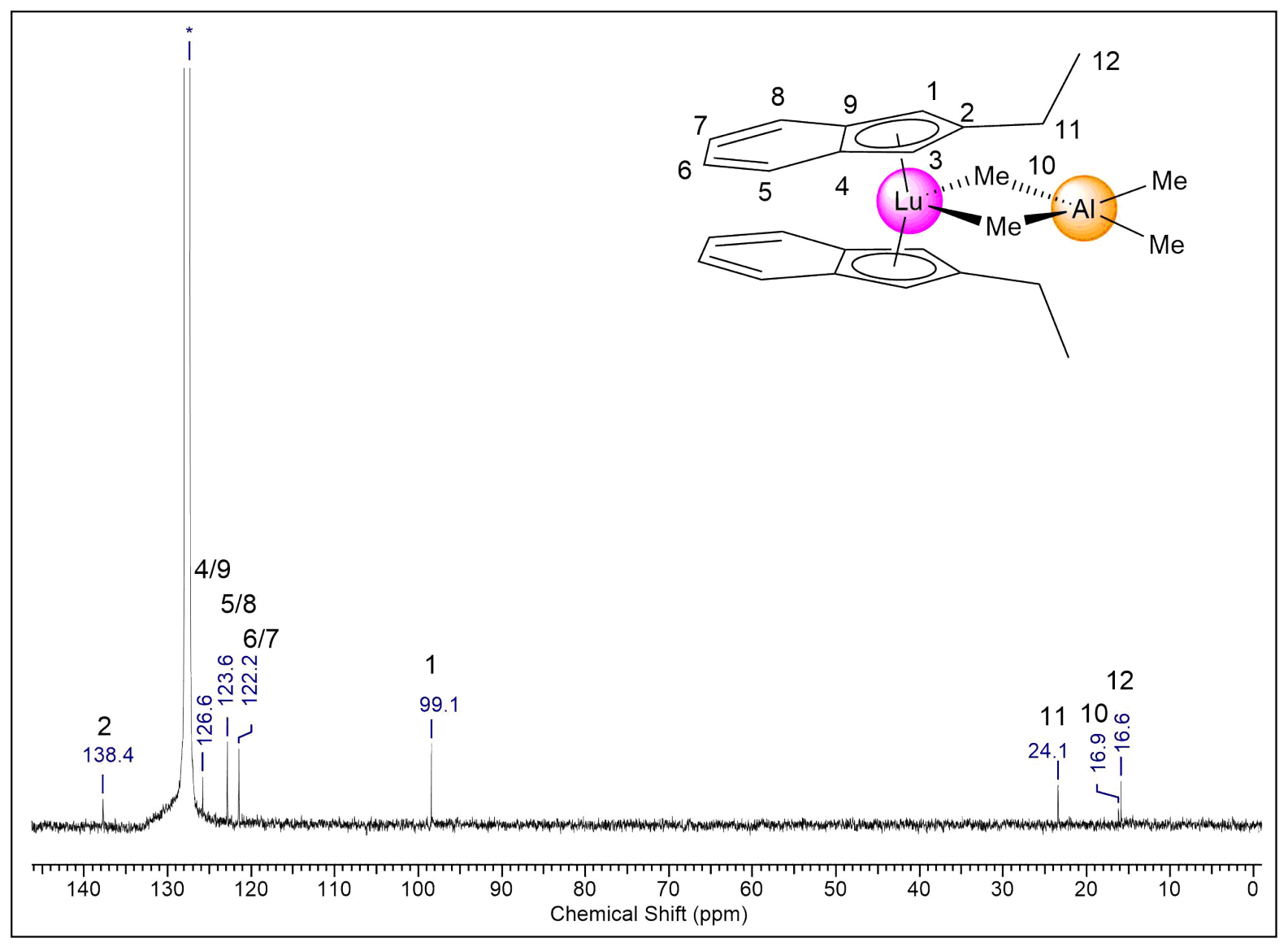

Figure S22. ${ }^{13} \mathrm{C}\left\{{ }^{1} \mathrm{H}\right\}$ NMR spectrum $(101 \mathrm{MHz})$ of $\left(\mathrm{Ind}^{\mathrm{Et}}\right)_{2} \mathrm{Lu}\left(\mathrm{AlMe}_{4}\right)(\mathbf{2 b})$ in $\mathrm{C}_{6} \mathrm{D}_{6}$ at $26{ }^{\circ} \mathrm{C}$. 



Figure S23. ${ }^{1} \mathrm{H}^{13} \mathrm{C}-\mathrm{HSQC}$ NMR spectrum $(400 / 101 \mathrm{MHz})$ of $\left(\mathrm{Ind}^{\mathrm{Et}}\right)_{2} \mathrm{La}\left(\mathrm{AlMe}_{4}\right)(\mathbf{2 b})$ in $\mathrm{C}_{6} \mathrm{D}_{6}$ at $26{ }^{\circ} \mathrm{C}$, including enlarged sections. 



Figure S24. ${ }^{1} \mathrm{H}^{1} \mathrm{H}-\mathrm{COSY}$ NMR spectrum $(400 / 400 \mathrm{MHz})$ of $\left(\mathrm{Ind}^{\mathrm{Et}}\right)_{2} \mathrm{Lu}\left(\mathrm{AlMe}_{4}\right)(\mathbf{2 b})$ in $\mathrm{C}_{6} \mathrm{D}_{6}$ at $26{ }^{\circ} \mathrm{C}$, including enlarged sections. 


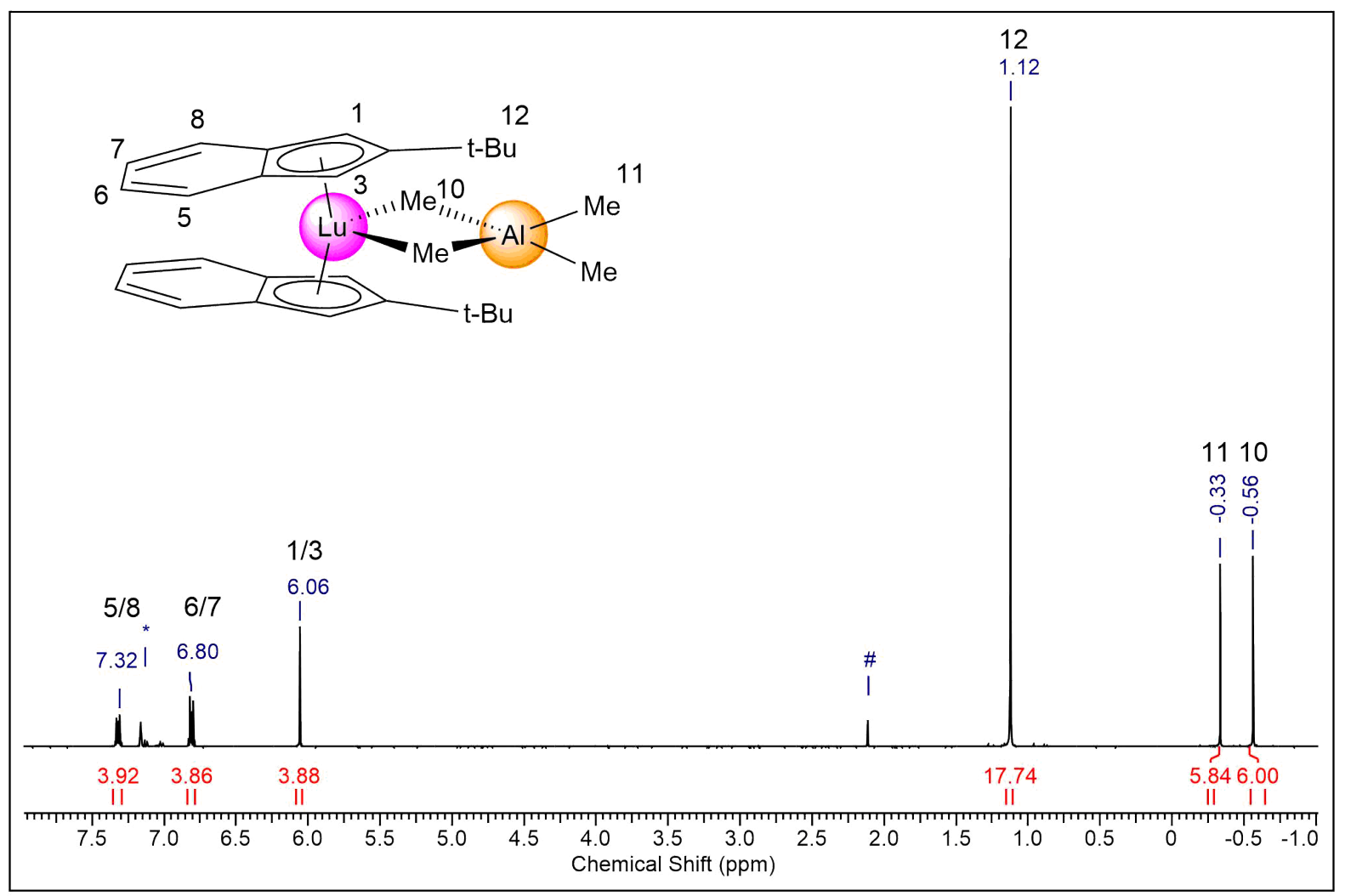

Figure S25. ${ }^{1} \mathrm{H}$ NMR spectrum $(400 \mathrm{MHz})$ of $\left(\mathrm{Ind}^{t \mathrm{Bu}}\right)_{2} \mathrm{Lu}\left(\mathrm{AlMe}_{4}\right)(\mathbf{2 c})$ in $\mathrm{C}_{6} \mathrm{D}_{6}$ at $26{ }^{\circ} \mathrm{C}$. Residual toluene marked with \#.

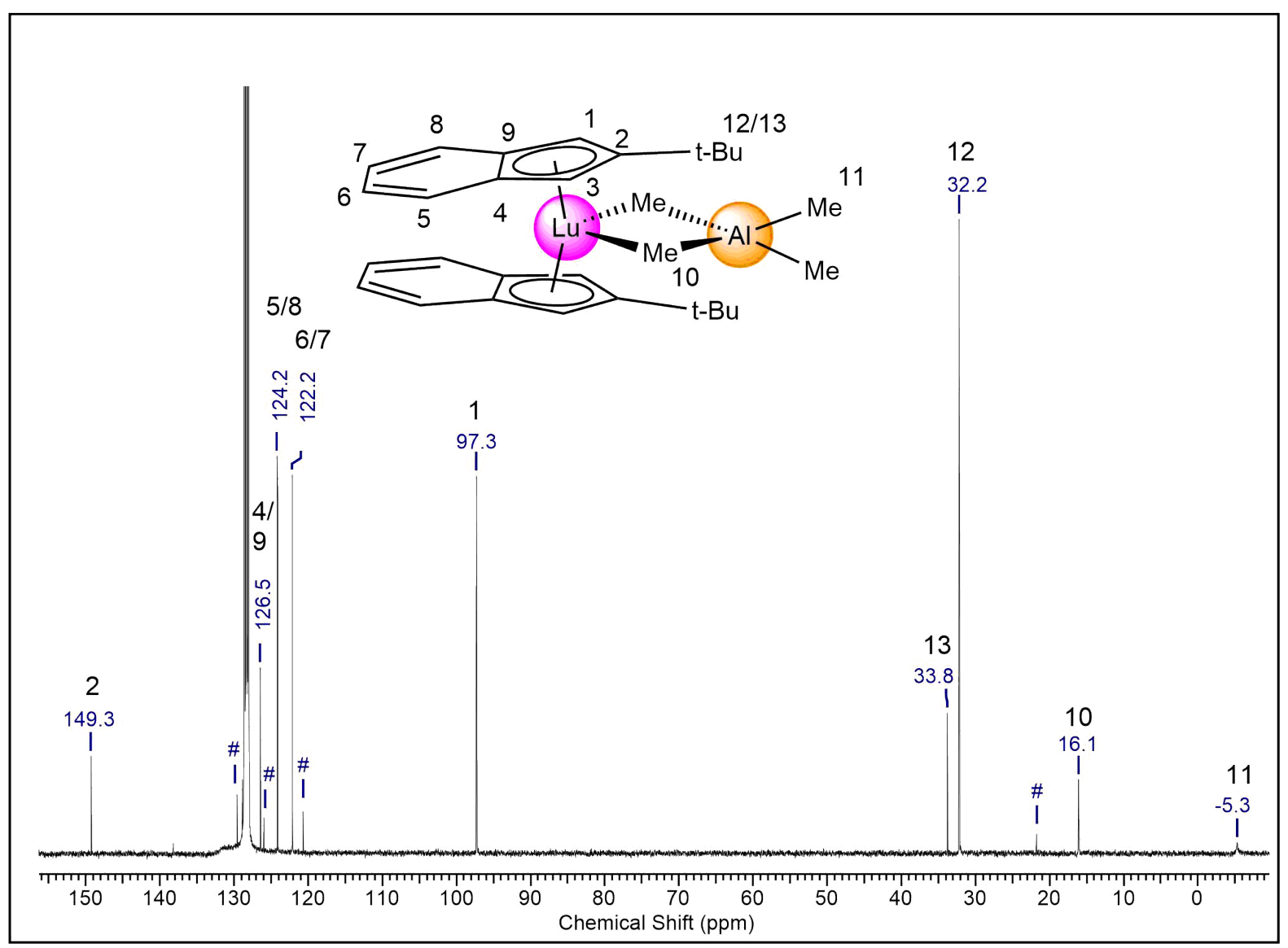

Figure S26. ${ }^{13} \mathrm{C}\left\{{ }^{1} \mathrm{H}\right\}$ NMR spectrum $(101 \mathrm{MHz})$ of $\left(\mathrm{Ind}^{\mathrm{tBu}}\right)_{2} \mathrm{Lu}\left(\mathrm{AlMe}_{4}\right)(\mathbf{2 c})$ in $\mathrm{C}_{6} \mathrm{D}_{6}$ at $26{ }^{\circ} \mathrm{C}$. Residual toluene marked with \#. 

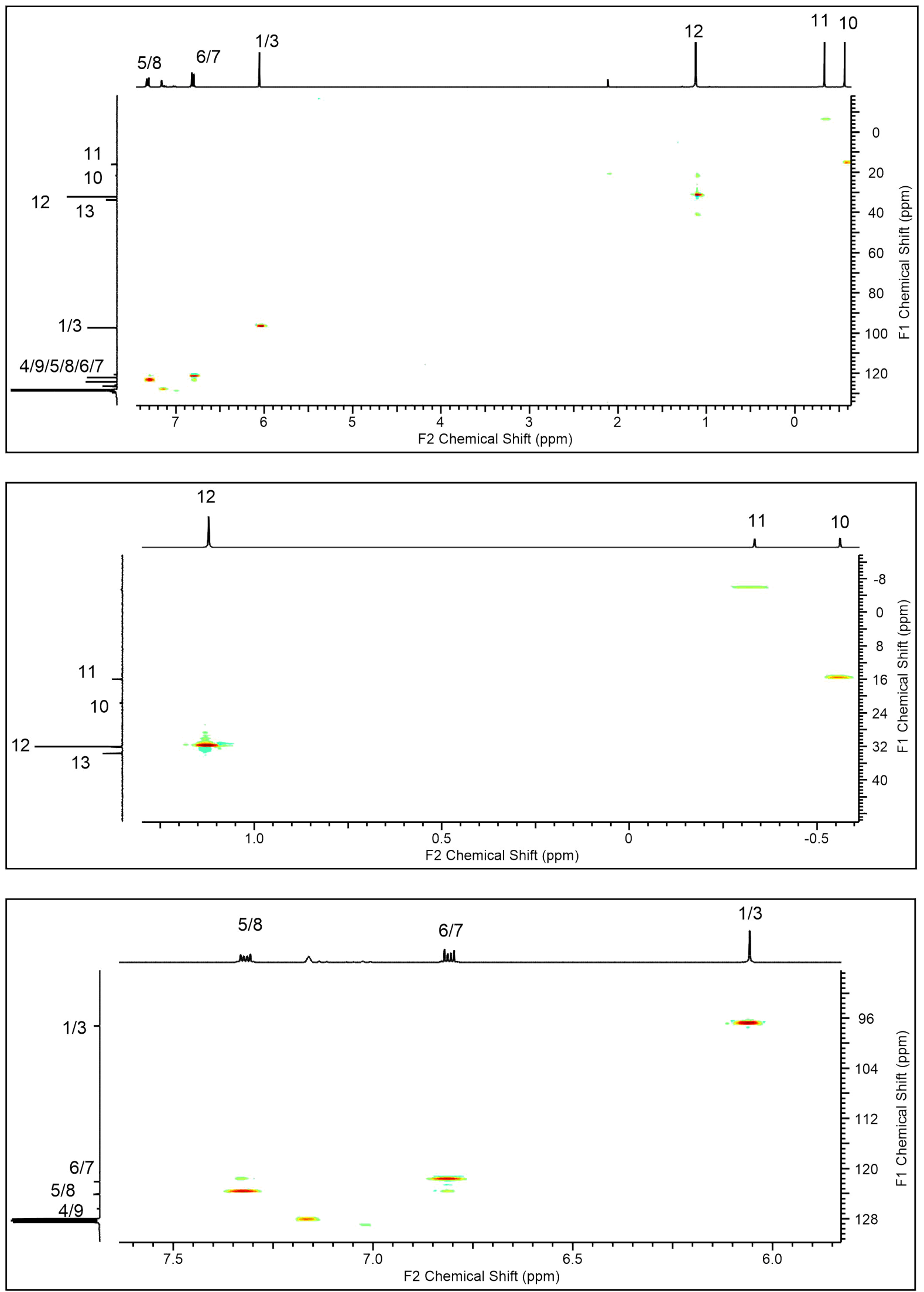

Figure S27. Section of ${ }^{1} \mathrm{H}^{13} \mathrm{C}-\mathrm{HSQC}$ NMR spectrum $(400 / 101 \mathrm{MHz})$ of $\left(\mathrm{Ind}^{t B u}\right)_{2} \mathrm{Lu}\left(\mathrm{AlMe}_{4}\right)(2 \mathrm{c})$ in $\mathrm{C}_{6} \mathrm{D}_{6}$ at $26^{\circ} \mathrm{C}$, including enlarged sections. 

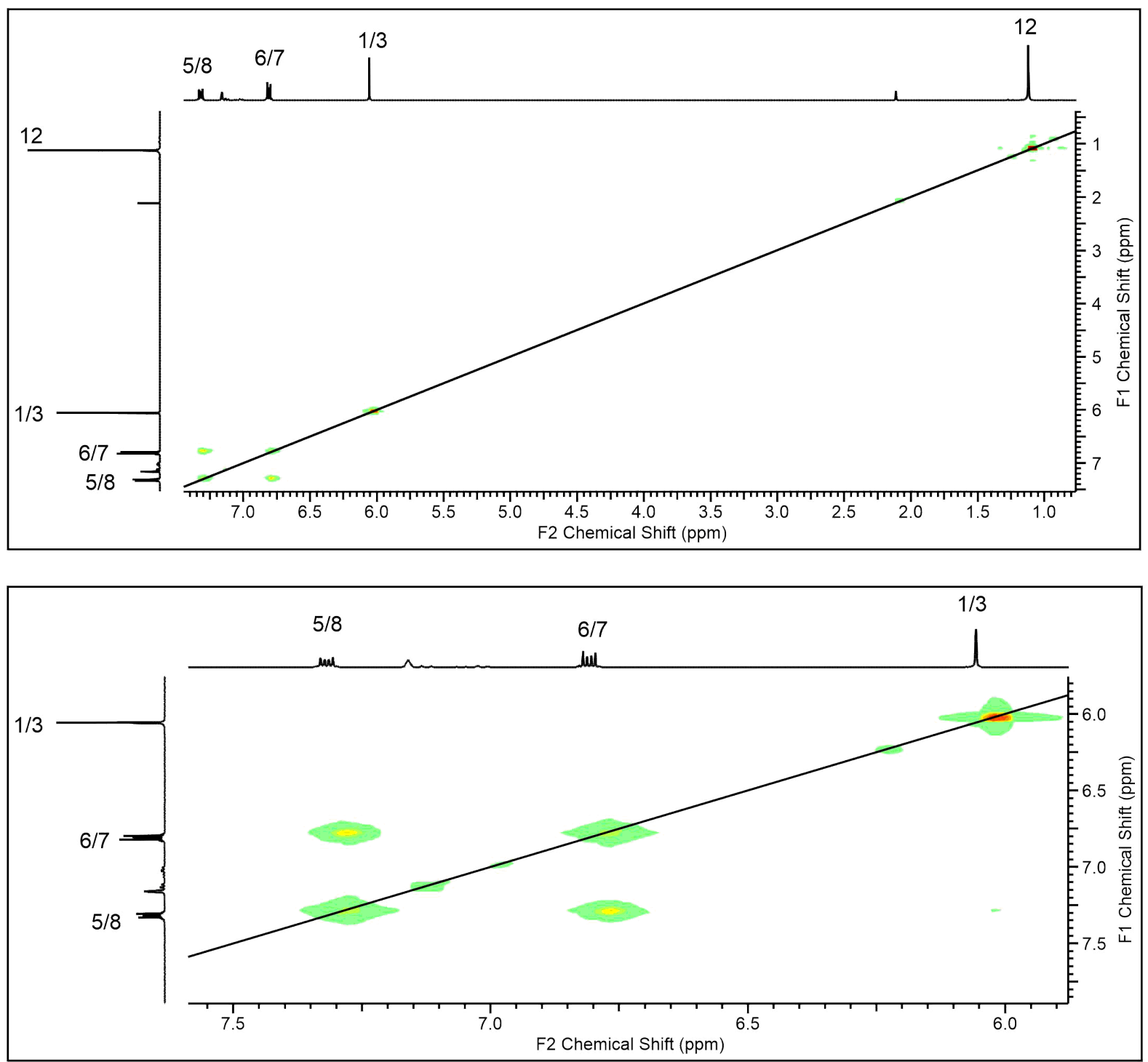

Figure S28. ${ }^{1} \mathrm{H}^{1} \mathrm{H}-\mathrm{COSY}$ NMR spectrum $(400 / 400 \mathrm{MHz})$ of $\left(\operatorname{Ind}^{t B u}\right)_{2} \mathrm{Lu}\left(\mathrm{AlMe}_{4}\right)(2 \mathrm{c})$ in $\mathrm{C}_{6} \mathrm{D}_{6}$ at $26^{\circ} \mathrm{C}$, including an enlarged section. 


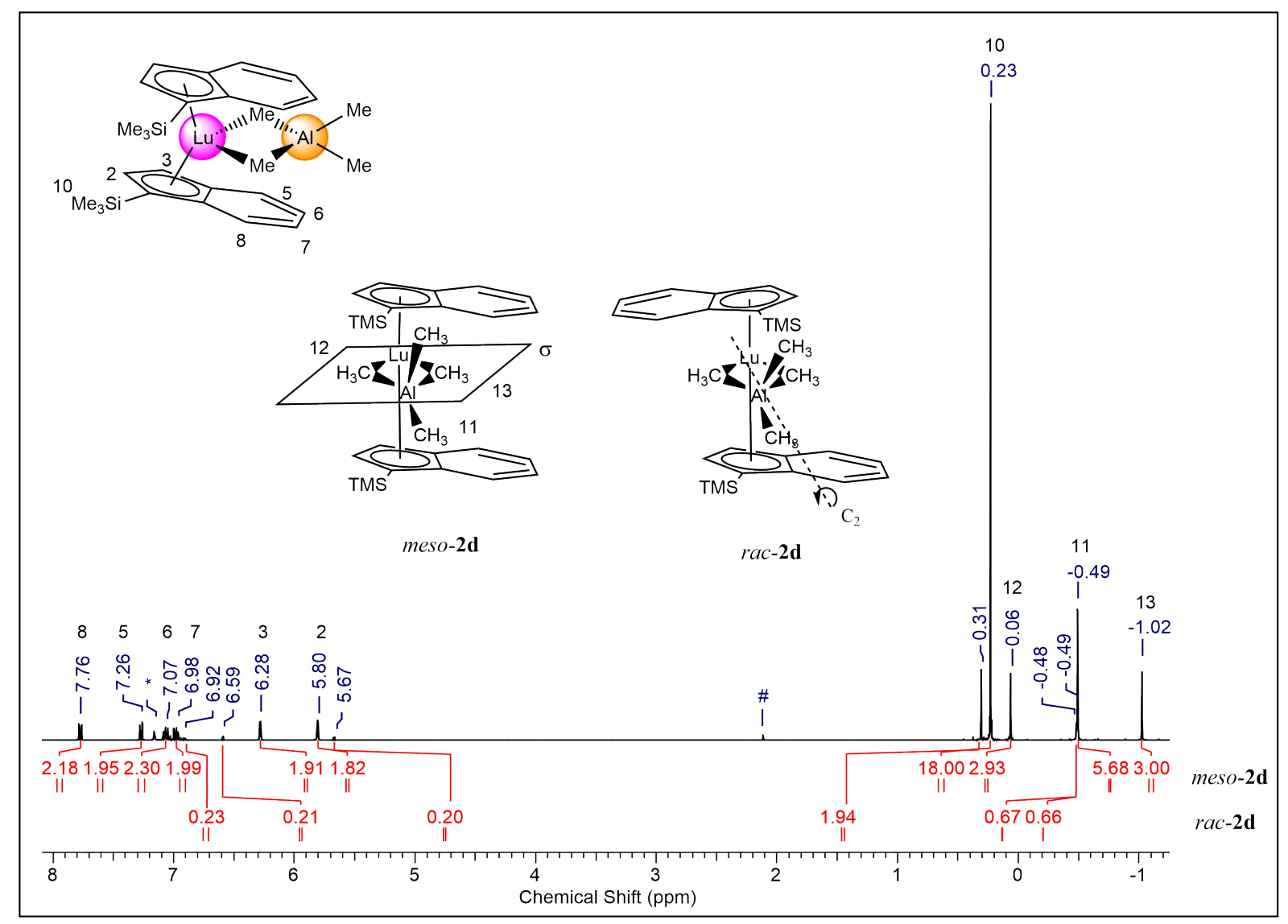

Figure S29. ${ }^{1} \mathrm{H}$ NMR spectrum $(400 \mathrm{MHz})$ of $\left(\mathrm{Ind}^{\mathrm{Si}}\right)_{2} \mathrm{Lu}\left(\mathrm{AlMe}_{4}\right)(\mathbf{2 d})$ in $\mathrm{C}_{6} \mathrm{D}_{6}$ at $26{ }^{\circ} \mathrm{C}$.

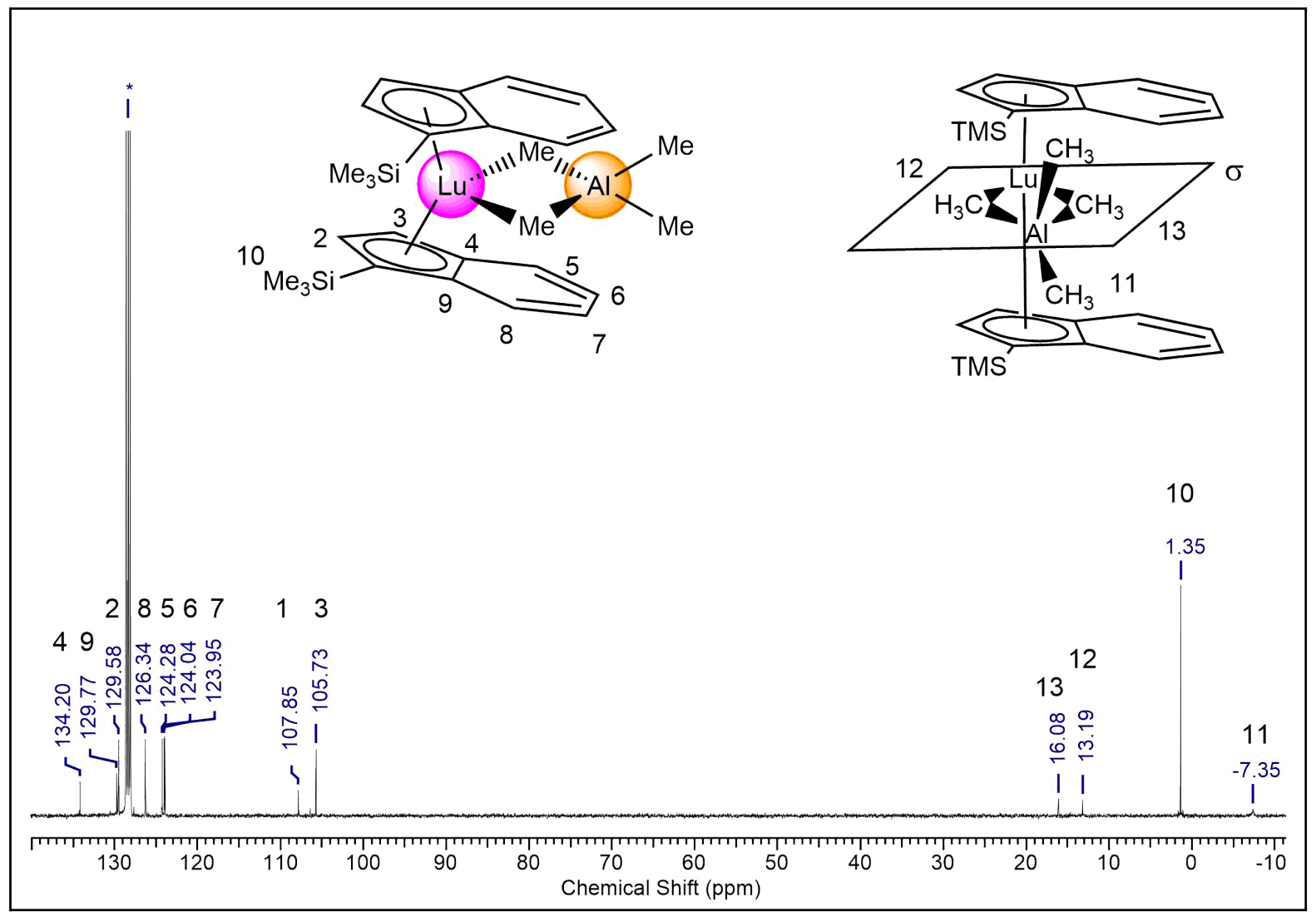

Figure S30. ${ }^{13} \mathrm{C}\left\{{ }^{1} \mathrm{H}\right\}$ NMR spectrum $(101 \mathrm{MHz})$ of $\left(\mathrm{Ind}^{\mathrm{Si}}\right)_{2} \mathrm{Lu}\left(\mathrm{AlMe}_{4}\right)(\mathbf{2 d})$ in $\mathrm{C}_{6} \mathrm{D}_{6}$ at $26{ }^{\circ} \mathrm{C}$. 

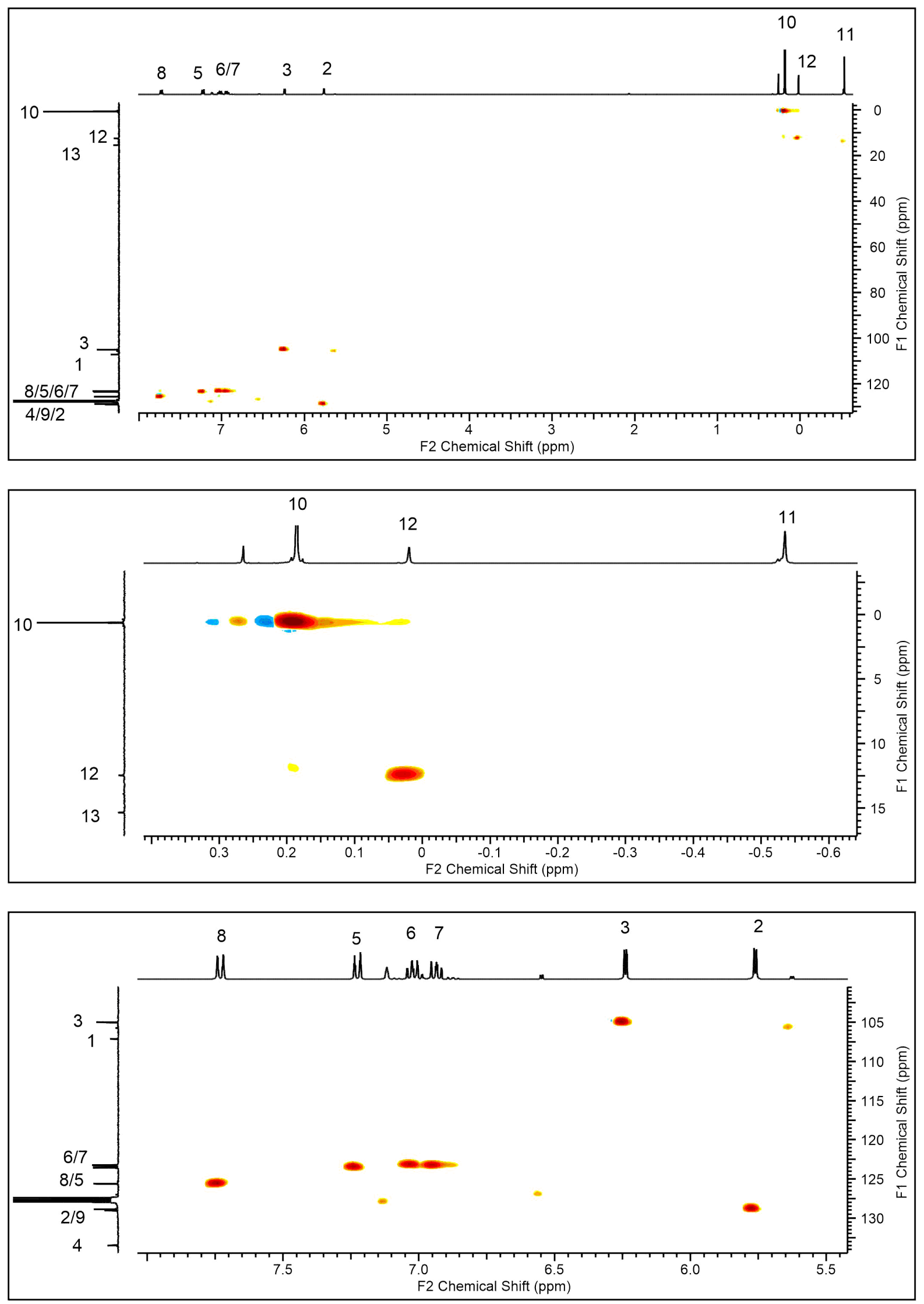

Figure S31. ${ }^{1} \mathrm{H}^{13} \mathrm{C}-\mathrm{HSQC}$ NMR spectrum $(400 / 101 \mathrm{MHz})$ of $\left(\mathrm{Ind}^{\mathrm{Si}}\right)_{2} \mathrm{Lu}\left(\mathrm{AlMe}_{4}\right)(\mathbf{2 d})$ in $\mathrm{C}_{6} \mathrm{D}_{6}$ at $26{ }^{\circ} \mathrm{C}$, including enlarged sections. 

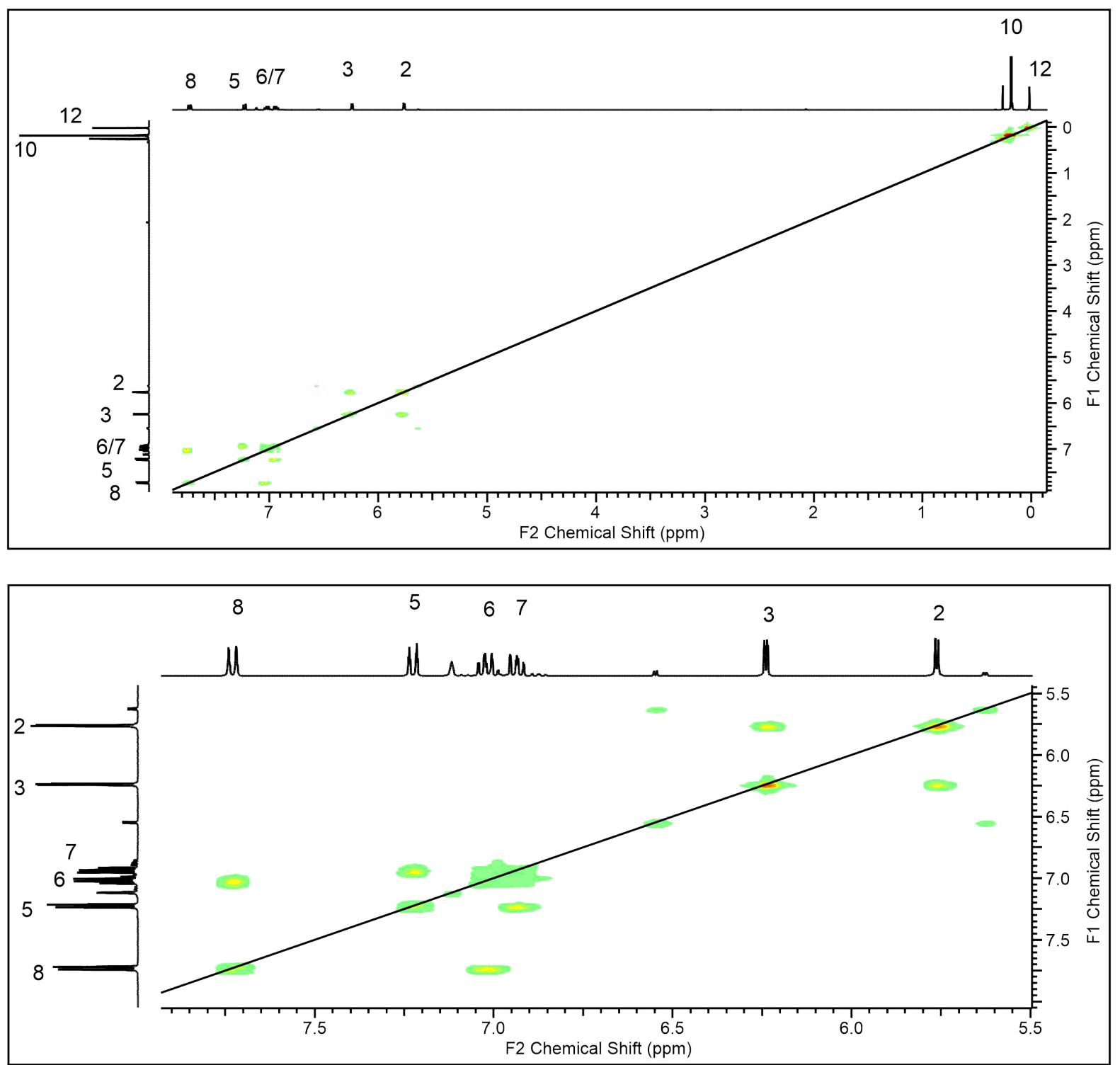

Figure S32. ${ }^{1} \mathrm{H}^{1} \mathrm{H}-\mathrm{COSY}$ NMR spectrum $(400 / 400 \mathrm{MHz})$ of $\left(\mathrm{Ind}^{\mathrm{Si}}\right)_{2} \mathrm{Lu}\left(\mathrm{AlMe}_{4}\right)(\mathbf{2 d})$ in $\mathrm{C}_{6} \mathrm{D}_{6}$ at $26^{\circ} \mathrm{C}$, including an enlarged section. 


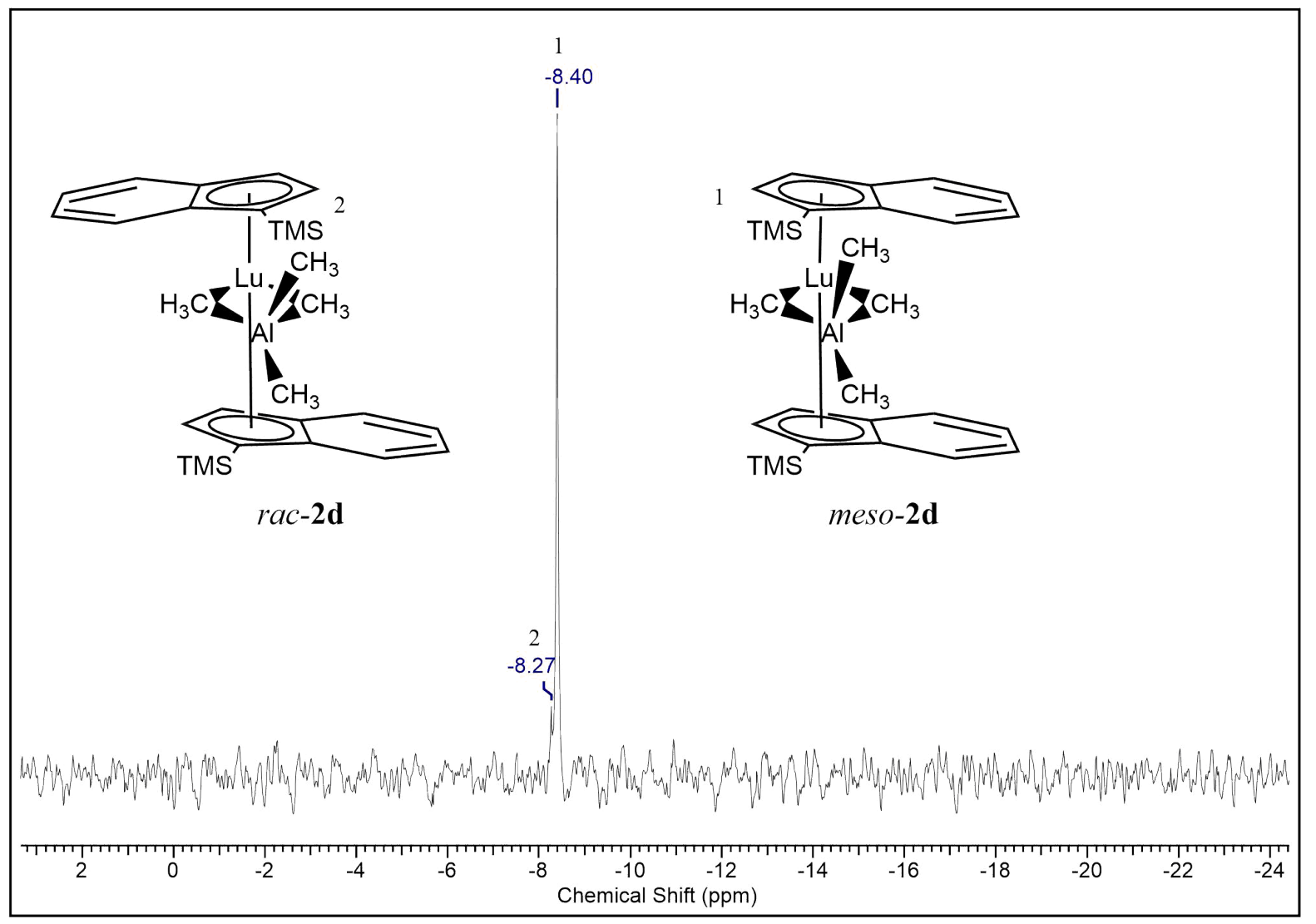

Figure S33. ${ }^{29} \mathrm{Si}^{-1} \mathrm{H}$ DEPT45 NMR spectrum $(50 \mathrm{MHz})$ of $\left(\mathrm{Ind}^{\mathrm{Si}}\right)_{2} \mathrm{Lu}\left(\mathrm{AlMe}_{4}\right)(\mathbf{2 d})$ in $\mathrm{C}_{6} \mathrm{D}_{6}$ at $26{ }^{\circ} \mathrm{C}$.

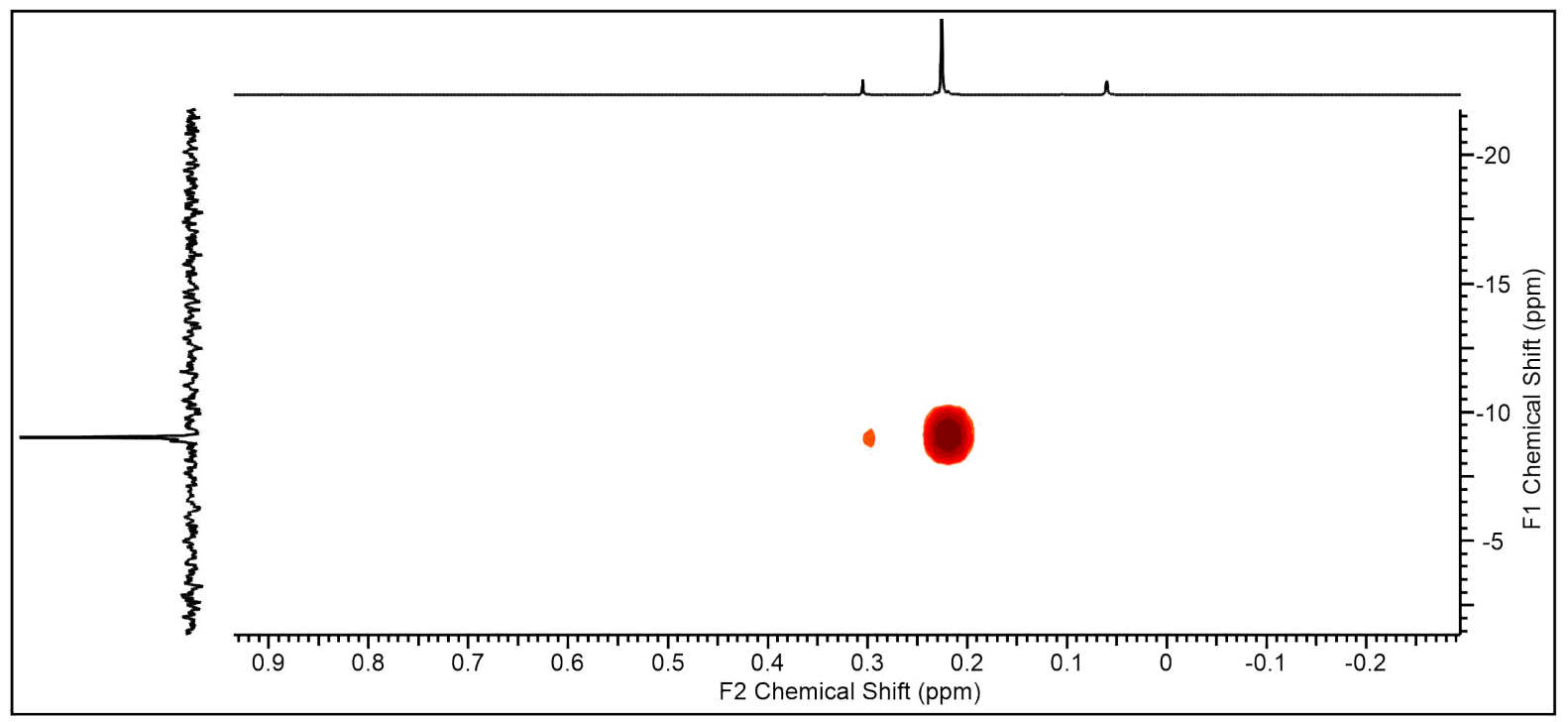

Figure S34. ${ }^{1} \mathrm{H}^{29} \mathrm{Si}-\mathrm{HMBC}$ NMR spectrum $(500 / 99 \mathrm{MHz})$ of $\left(\mathrm{Ind}^{\mathrm{Si}}\right)_{2} \mathrm{Lu}\left(\mathrm{AlMe}_{4}\right)(\mathbf{2 d})$ in $\mathrm{C}_{6} \mathrm{D}_{6}$ at $26{ }^{\circ} \mathrm{C}$. 


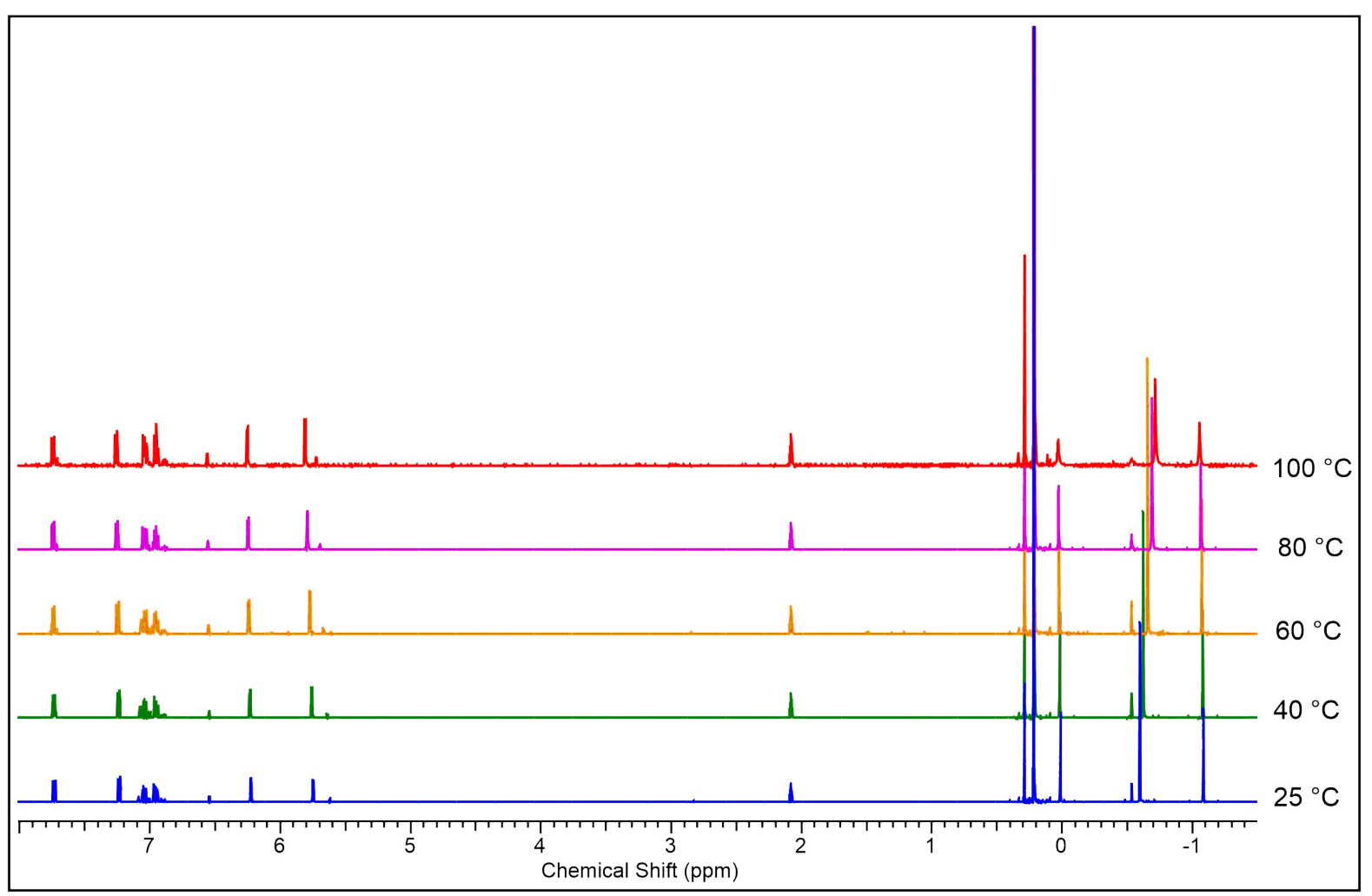

Figure S35. VT ${ }^{1} \mathrm{H}$ NMR spectrum $(500 \mathrm{MHz})$ of $\left(\mathrm{Ind}^{\mathrm{Si}}\right)_{2} \mathrm{Lu}\left(\mathrm{AlMe}_{4}\right)(\mathbf{2 d})$ in tolune- $d 8$. From $25^{\circ} \mathrm{C}$ to $100{ }^{\circ} \mathrm{C}$. 


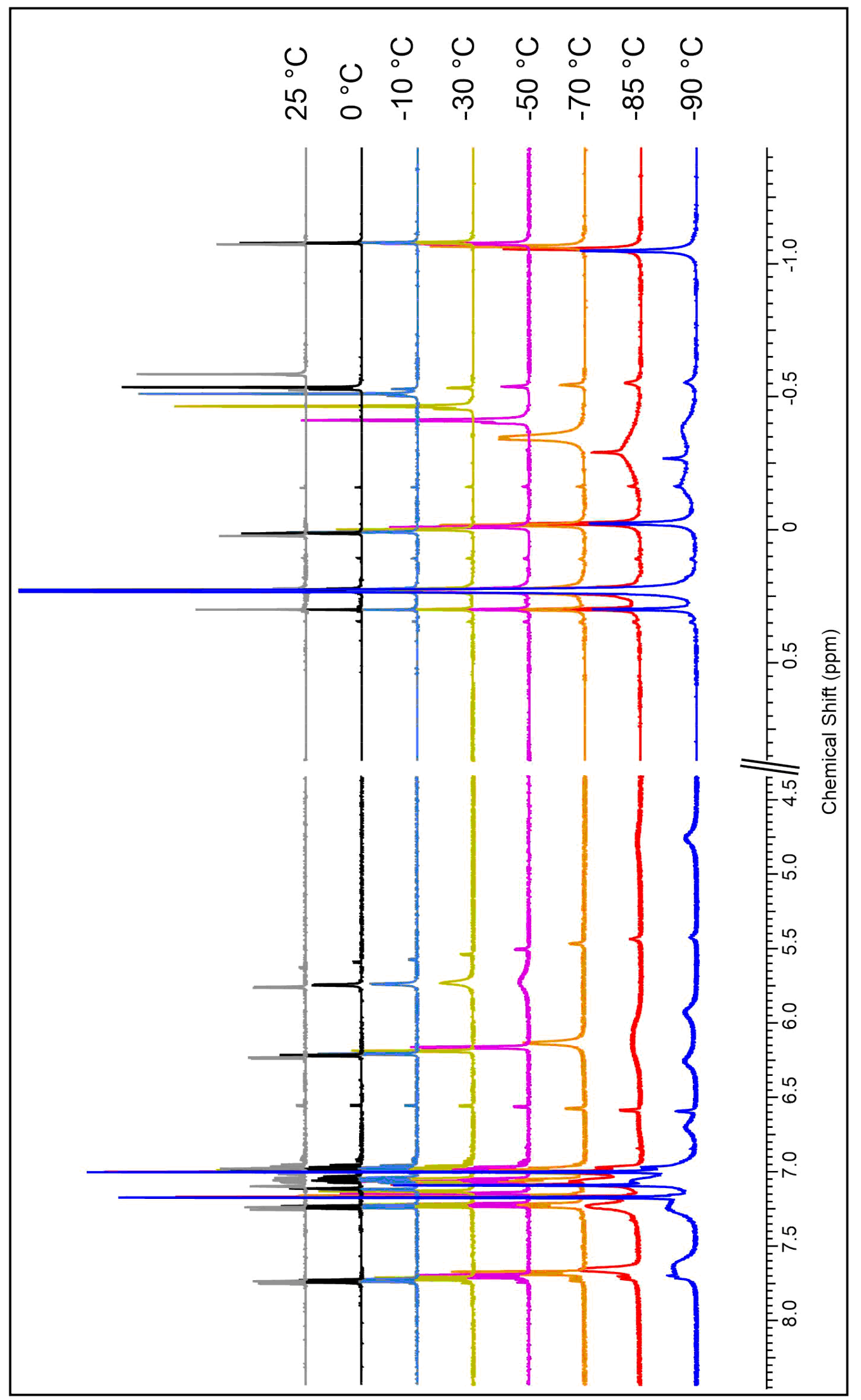

Figure S36. VT ${ }^{1} \mathrm{H}$ NMR spectrum $(500 \mathrm{MHz})$ of $\left(\mathrm{Ind}^{\mathrm{Si}}\right)_{2} \mathrm{Lu}\left(\mathrm{AlMe}_{4}\right)(\mathbf{2 d})$ in tolune- $d 8$. From $-90{ }^{\circ} \mathrm{C}$ to $25^{\circ} \mathrm{C}$. 


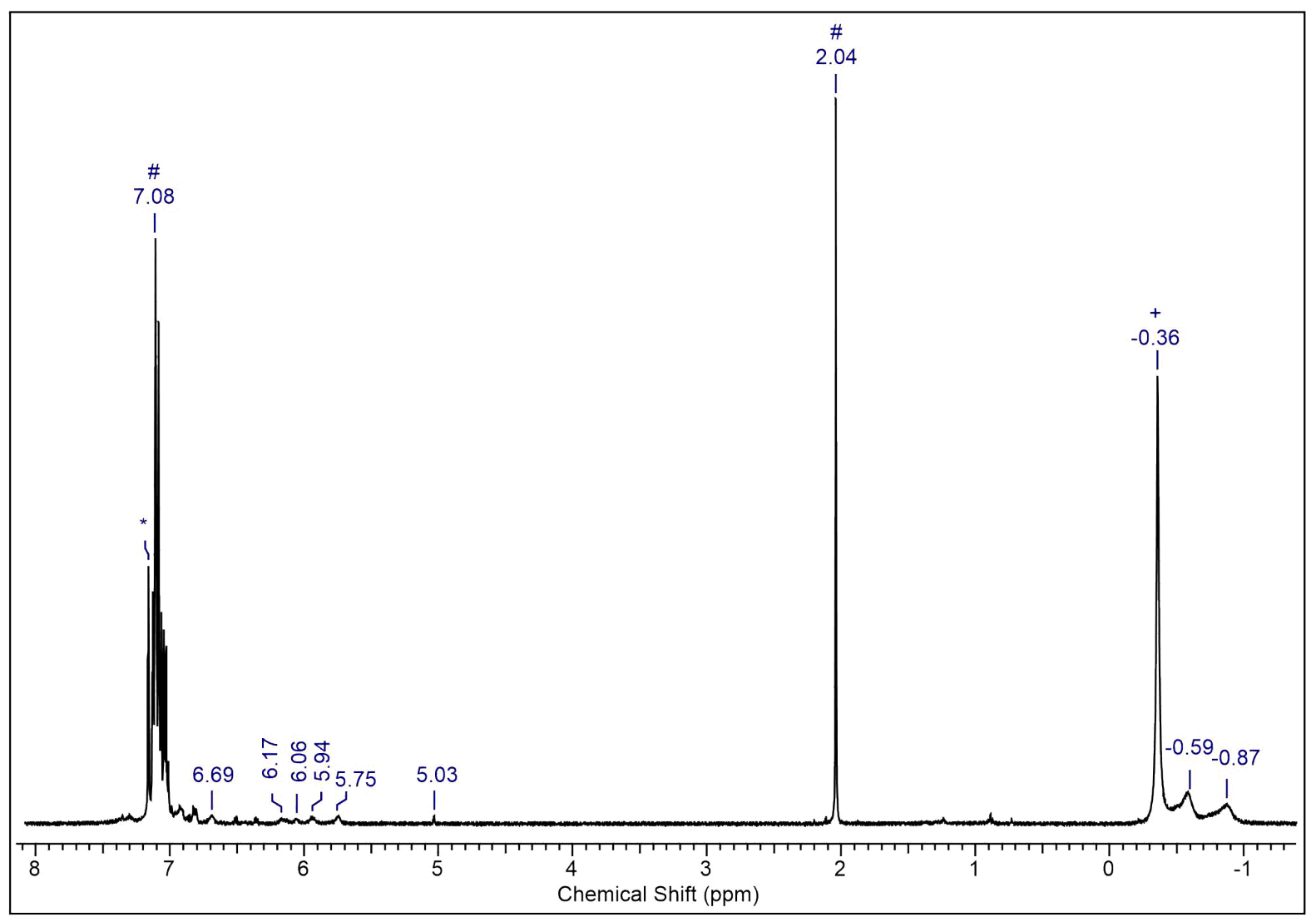

Figure S37. ${ }^{1} \mathrm{H}$ NMR spectrum $(400 \mathrm{MHz})$ of the reaction of (Ind)La(AlMe $)_{2}(\mathbf{1 a})$ with $\left[\mathrm{Ph}_{3} \mathrm{C}\right]\left[\mathrm{B}\left(\mathrm{C}_{6} \mathrm{~F}_{5}\right)_{4}\right]$ in $\mathrm{C}_{6} \mathrm{D}_{6}$ at $26{ }^{\circ} \mathrm{C}$. $\mathrm{Ph}_{3} \mathrm{CMe}$ is marked with \#; free trimethylaluminum is marked +.

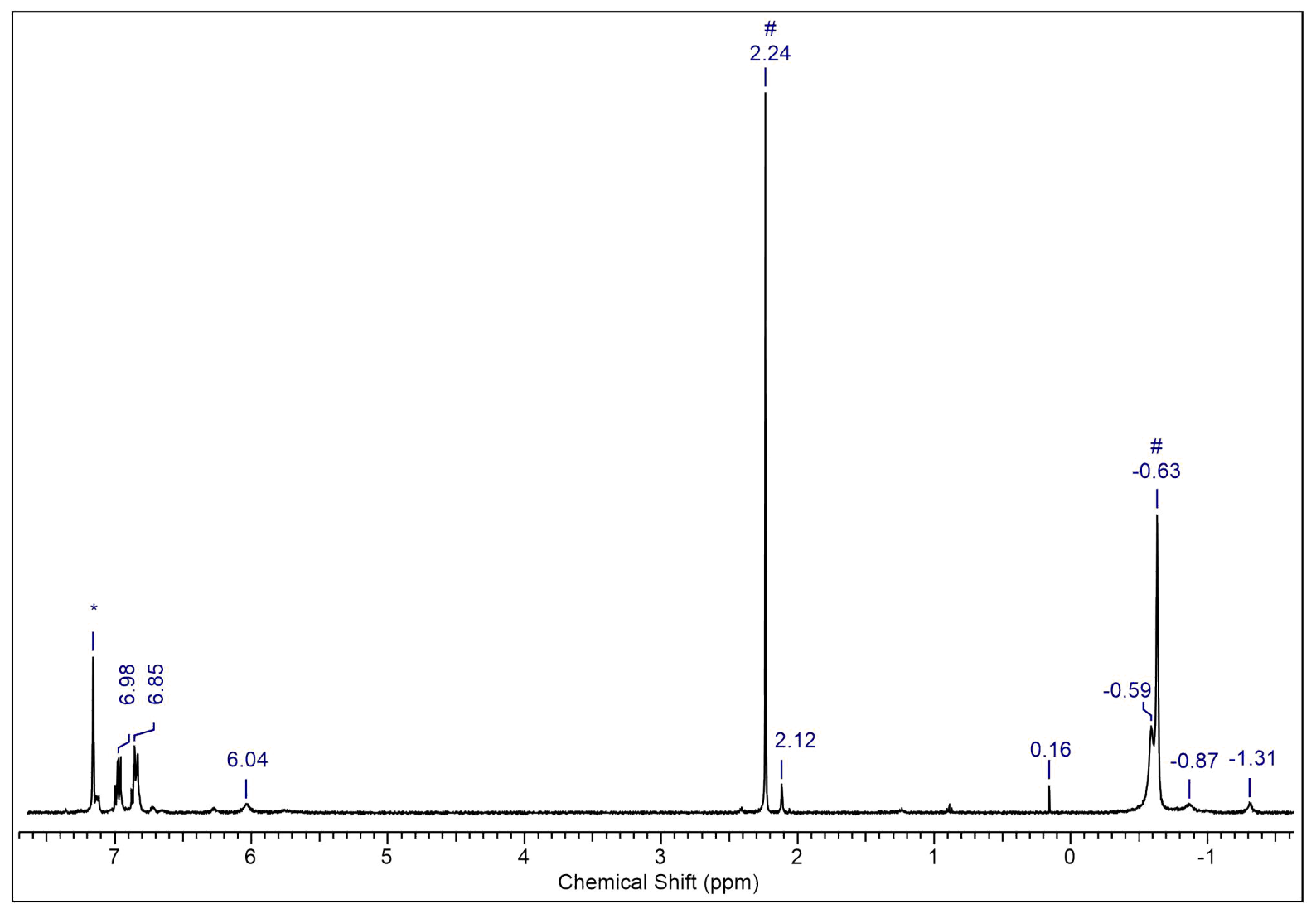

Figure S38. ${ }^{1} \mathrm{H}$ NMR spectrum $(400 \mathrm{MHz})$ of the reaction of (Ind)La(AlMe $)_{2}(\mathbf{1 a})$ with $\left[\mathrm{PhNMe}_{2} \mathrm{H}\right]\left[\mathrm{B}_{(}\left(\mathrm{C}_{6} \mathrm{~F}_{5}\right)_{4}\right]$ in $\mathrm{C}_{6} \mathrm{D}_{6}$ at $26^{\circ} \mathrm{C} . \mathrm{PhNMe}_{2}\left(\mathrm{AlMe}_{3}\right)$ is marked with \#. 


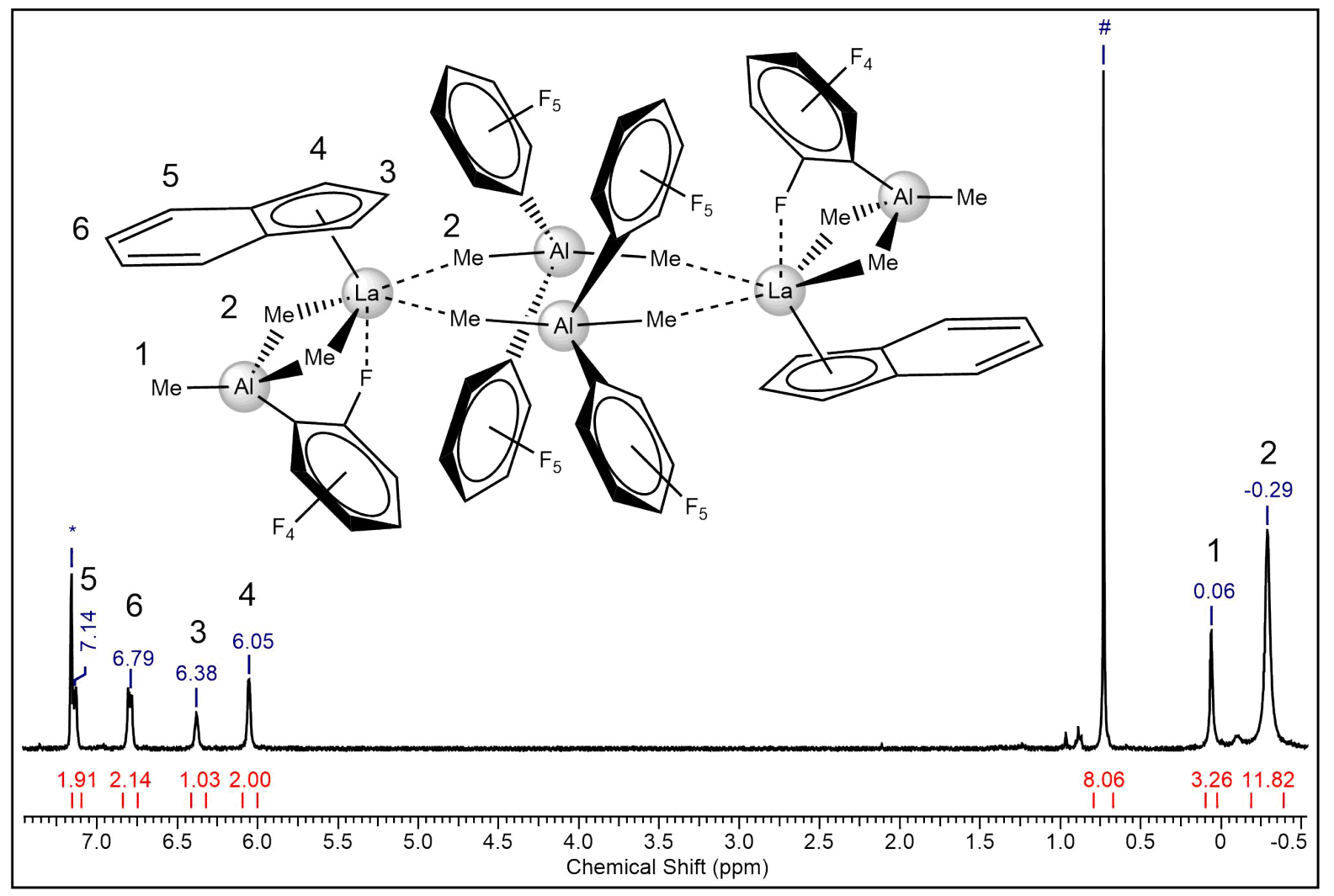

Figure S39. ${ }^{1} \mathrm{H}$ NMR spectrum $\left(400 \mathrm{MHz}\right.$ ) of the reaction of (Ind)La( $\left(\mathrm{AlMe}_{4}\right)_{2}$ with $\mathrm{B}\left(\mathrm{C}_{6} \mathrm{~F}_{5}\right)_{3}$ in $\mathrm{C}_{6} \mathrm{D}_{6}$ at $26{ }^{\circ} \mathrm{C}$. $\mathrm{BMe}_{3}$ is marked with \#.



Figure S40. ${ }^{11} \mathrm{~B}$ NMR spectrum $\left(400 \mathrm{MHz}\right.$ ) of the reaction of (Ind) $\mathrm{La}\left(\mathrm{AlMe}_{4}\right)_{2}$ with $\mathrm{B}\left(\mathrm{C}_{6} \mathrm{~F}_{5}\right)_{3}$ in $\mathrm{C}_{6} \mathrm{D}_{6}$ at $26{ }^{\circ} \mathrm{C}$ showing the formation of $\mathrm{BMe}_{3}$. 




Figure S41. Molecular structure of $\left(\mathrm{Ind} \mathrm{Et}^{\mathrm{Et}} \mathrm{La}\left(\mathrm{AIMe}_{4}\right)_{2}\right.$ (1b). Hydrogen atoms are omitted for clarity. Atomic displacement parameters set at the 50\% probability level. Selected bond lengths $[\AA]$ and angles $\left[^{\circ}\right]$ : La1-C1 2.781(5), La1-C2 2.836(4), La1-C3 2.792(4), La1-C4 2.815(5), La1-C9 2.803(5), La1-C10 2.967(5), La1-C11 2.757(5), La1-C14 2.694(5), La1-C15

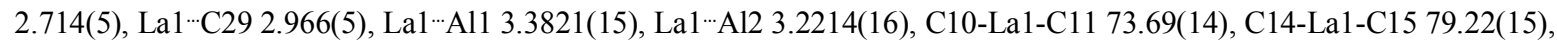
La2-C18 2.796(5), La2-C19 2.885(5), La2-C20 2.798(4), La2-C21 2.794(5), La2-C26 2.797(5), La2-C27 2.912(5), La2-

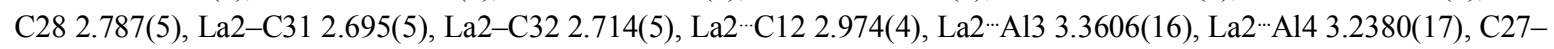
La2-C28 74.55(15), C31-La2-C32 78.77(15). 
Table S1. Crystallographic Data for Compounds 1a, 1b, 1c, and 1d

\begin{tabular}{|c|c|c|c|c|}
\hline & $1 \mathbf{a}$ & $1 \mathbf{b}$ & $1 \mathrm{c}$ & 1d \\
\hline CCDC number & 1915979 & 1915982 & 1915976 & 1915977 \\
\hline formula & $\mathrm{C}_{20.5} \mathrm{H}_{35} \mathrm{Al}_{2} \mathrm{La}$ & $\mathrm{C}_{19} \mathrm{H}_{35} \mathrm{Al}_{2} \mathrm{La}$ & $\mathrm{C}_{21} \mathrm{H}_{39} \mathrm{Al}_{2} \mathrm{La}$ & $\mathrm{C}_{20} \mathrm{H}_{39} \mathrm{Al}_{2} \mathrm{LaSi}$ \\
\hline $\mathrm{M}\left[\mathrm{g} \cdot \mathrm{mol}^{-1}\right]$ & 474.35 & 456.34 & 484.39 & 500.47 \\
\hline Color & colorless & colorless & colorless & colorless \\
\hline $\begin{array}{c}\text { Crystal } \\
\text { dimensions }[\mathrm{mm}]\end{array}$ & $\begin{array}{c}0.234 \times 0.170 \mathrm{x} \\
0.096\end{array}$ & $\begin{array}{c}0.175 \times 0.161 \times \\
0.085\end{array}$ & $\begin{array}{c}0.528 \times 0.457 \times \\
0.244\end{array}$ & $\begin{array}{c}0.480 \times 0.244 \times \\
0.188\end{array}$ \\
\hline Crystal system & monoclinic & orthorhombic & trigonal & triclinic \\
\hline space group & $\mathrm{P} 2{ }_{1} / \mathrm{c}$ & $\mathrm{Pca} 2_{1}$ & $\mathrm{P} 3_{1}$ & $\mathrm{P} \overline{1}$ \\
\hline $\mathrm{a}[\AA]$ & $19.459(5)$ & $19.545(3)$ & $9.7198(5)$ & $9.4353(5)$ \\
\hline $\mathrm{b}[\AA]$ & $11.634(3)$ & $10.1865(17)$ & $9.7198(5)$ & $9.6041(5)$ \\
\hline c $[\AA]$ & $21.681(6)$ & $22.334(4)$ & $22.1290(10)$ & $15.4562(8)$ \\
\hline$\alpha\left[^{\circ}\right]$ & 90 & 90 & 90 & $93.888(2)$ \\
\hline$\beta\left[^{\circ}\right]$ & $105.935(4)$ & 90 & 90 & $103.960(2)$ \\
\hline$\gamma\left[^{\circ}\right]$ & 90 & 90 & 120 & $116.010(2)$ \\
\hline $\mathrm{V}\left[\AA^{3}\right]$ & $4720(2)$ & $4446.7(13)$ & $1810.5(2)$ & 1197.13 \\
\hline $\mathrm{Z}$ & 8 & 8 & 3 & 2 \\
\hline $\mathrm{T}[\mathrm{K}]$ & $155(2)$ & $150(2)$ & $103(2)$ & $100(2)$ \\
\hline$\rho_{\text {calcd }}\left[\mathrm{g} \cdot \mathrm{cm}^{-3}\right]$ & 1.335 & 1.363 & 1.333 & 1.388 \\
\hline$\mu\left[\mathrm{mm}^{-1}\right]$ & 1.884 & 1.997 & 1.843 & 1.908 \\
\hline $\mathrm{F}(000)$ & 1928 & 1856 & 744 & 512 \\
\hline Unique reflns & 13199 & 9267 & 7082 & 6161 \\
\hline Observed reflns & 74853 & 90976 & 25431 & 35570 \\
\hline $\mathrm{R} 1 / w \mathrm{R} 2(\mathrm{I}>2 \sigma)$ & $0.0314 / 0.0604$ & $0.0249 / 0.0450$ & $0.0163 / 0.0407$ & $0.0108 / 0.0288$ \\
\hline $\mathrm{R} 1 / w \mathrm{R} 2$ (all data) & $0.0501 / 0.0683$ & $0.0324 / 0.0472$ & $0.0164 / 0.0407$ & $0.0109 / 0.0289$ \\
\hline Goodness of fit & 1.022 & 1.038 & 1.077 & 1.080 \\
\hline
\end{tabular}


Table S2. Crystallographic Data for Compounds 2a, 2b, 2c, and 2d

\begin{tabular}{|c|c|c|c|c|}
\hline & $2 \mathbf{2 a}$ & $2 \mathbf{b}$ & $2 \mathrm{c}$ & $2 d$ \\
\hline CCDC number & 1915980 & 1915981 & 1915978 & 1915983 \\
\hline formula & $\mathrm{C}_{22} \mathrm{H}_{26} \mathrm{AlLu}$ & $\mathrm{C}_{26} \mathrm{H}_{34} \mathrm{AlLu}$ & $\mathrm{C}_{33.5} \mathrm{H}_{46} \mathrm{AlLu}$ & $\mathrm{C}_{28} \mathrm{H}_{42} \mathrm{AlLuSi}_{2}$ \\
\hline $\mathrm{M}\left[\mathrm{g} \cdot \mathrm{mol}^{-1}\right]$ & 492.38 & 548.48 & 650.65 & 636.74 \\
\hline Color & colorless & colorless & colorless & Colorless \\
\hline $\begin{array}{c}\text { Crystal } \\
\text { dimensions }[\mathrm{mm}]\end{array}$ & $\begin{array}{c}0.269 \times 0.159 \times \\
0.136\end{array}$ & $\begin{array}{c}0.331 \times 0.158 \times \\
0.153\end{array}$ & $\begin{array}{c}0.247 \times 0.126 \mathrm{x} \\
0.115\end{array}$ & $\begin{array}{c}0.495 \times 0.223 \times \\
0.220\end{array}$ \\
\hline Crystal system & monoclinic & monoclinic & triclinic & monoclinic \\
\hline space group & $\mathrm{Cc}$ & $\mathrm{P} 2{ }_{1} / \mathrm{c}$ & $\mathrm{P} \overline{1}$ & $\mathrm{P} 2{ }_{1} / \mathrm{c}$ \\
\hline $\mathrm{a}[\AA]$ & $14.297(2)$ & $13.9877(6)$ & $9.915(4)$ & $9.4943(13)$ \\
\hline $\mathrm{b}[\AA]$ & $14.304(2)$ & $18.5367(8)$ & $10.487(6)$ & $16.796(2)$ \\
\hline c $[\AA]$ & $19.222(3)$ & $9.3019(4)$ & $15.091(5)$ & $18.475(3)$ \\
\hline$\alpha\left[^{\circ}\right]$ & 90 & 90 & $75.814(11)$ & 90 \\
\hline$\beta\left[^{\circ}\right]$ & $97.286(2)$ & $103.447(2)$ & $81.200(6)$ & $98.438(4)$ \\
\hline$\gamma\left[{ }^{\circ}\right]$ & 90 & 90 & $77.448(11)$ & 90 \\
\hline $\mathrm{V}\left[\AA^{3}\right]$ & 3899.1(11) & $2345.73(18)$ & $1476.6(11)$ & $2914.2(7)$ \\
\hline $\mathrm{Z}$ & 8 & 4 & 2 & 4 \\
\hline $\mathrm{T}[\mathrm{K}]$ & $150(2)$ & $100(2)$ & $100(2)$ & $100(2)$ \\
\hline$\rho_{\text {calcd }}\left[\mathrm{g} \cdot \mathrm{cm}^{-3}\right]$ & 1.678 & 1.553 & 1.463 & 1.451 \\
\hline$\mu\left[\mathrm{mm}^{-1}\right]$ & 5.108 & 4.254 & 3.392 & 3.513 \\
\hline $\mathrm{F}(000)$ & 1936 & 1096 & 662 & 1288 \\
\hline Unique reflns & 9619 & 6586 & 8656 & 7837 \\
\hline Observed reflns & 29878 & 58663 & 63482 & 44485 \\
\hline $\mathrm{R} 1 / w \mathrm{R} 2(\mathrm{I}>2 \sigma)$ & $0.0221 / 0.0514$ & $0.0144 / 0.0342$ & $0.0204 / 0.0482$ & $0.0172 / 0.0415$ \\
\hline $\mathrm{R} 1 / w \mathrm{R} 2$ (all data) & $0.0227 / 0.0516$ & $0.0163 / 0.0350$ & $0.0228 / 0.0491$ & $0.0185 / 0.0420$ \\
\hline Goodness of fit & 1.092 & 0.982 & 1.106 & 1.075 \\
\hline
\end{tabular}




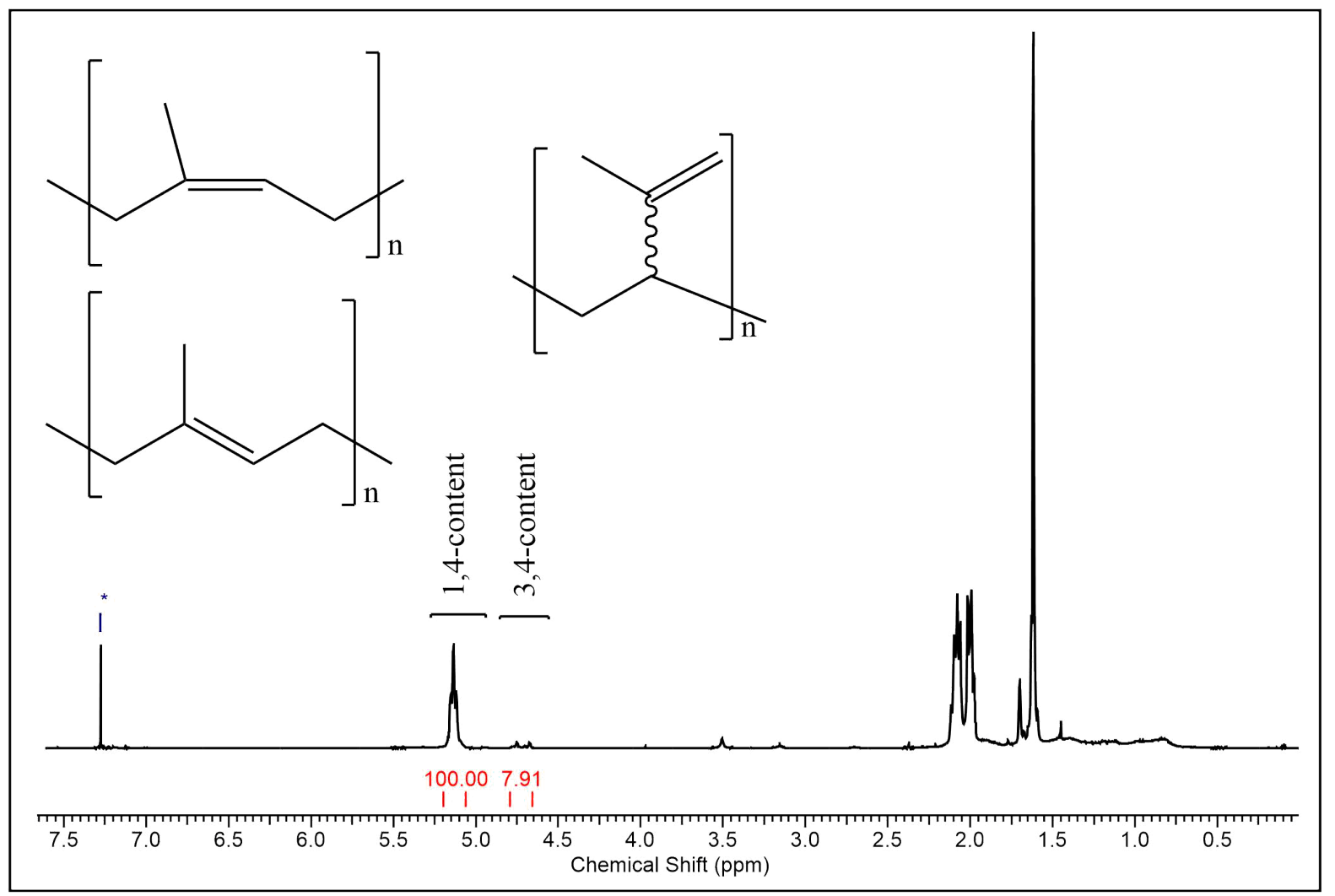

Figure S42. ${ }^{1} \mathrm{H}$ NMR spectrum (400 MHz) of polyisoprene (entry 4) in $\mathrm{CDCl}_{3}$ at $26{ }^{\circ} \mathrm{C}$.

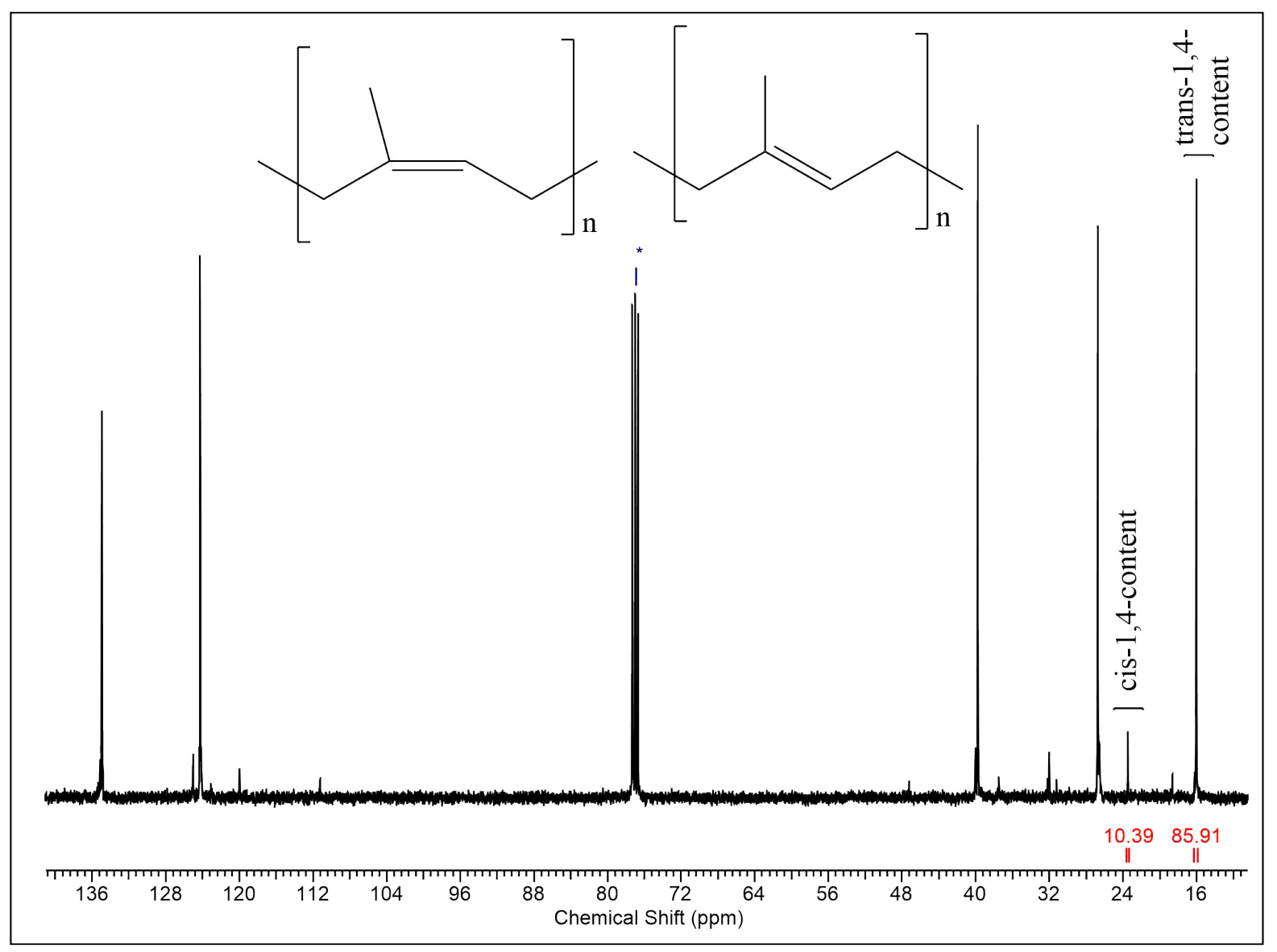

Figure S43. ${ }^{13} \mathrm{C}\left\{{ }^{1} \mathrm{H}\right\}$ NMR spectrum (101 MHz) of polyisoprene (entry 4) in $\mathrm{CDCl}_{3}$ at $26{ }^{\circ} \mathrm{C}$. 


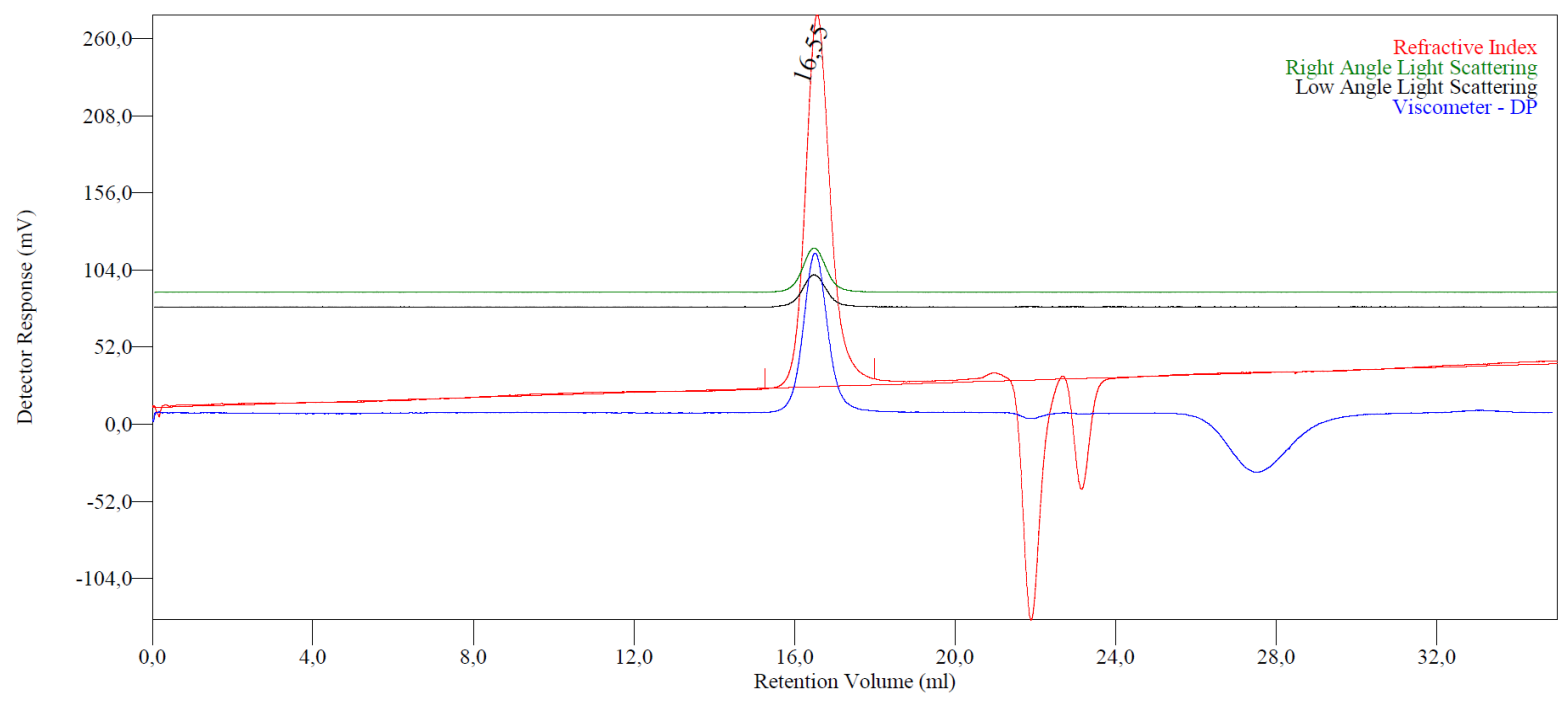

Figure S44. GPC curve of polyisoprene (entry 4).

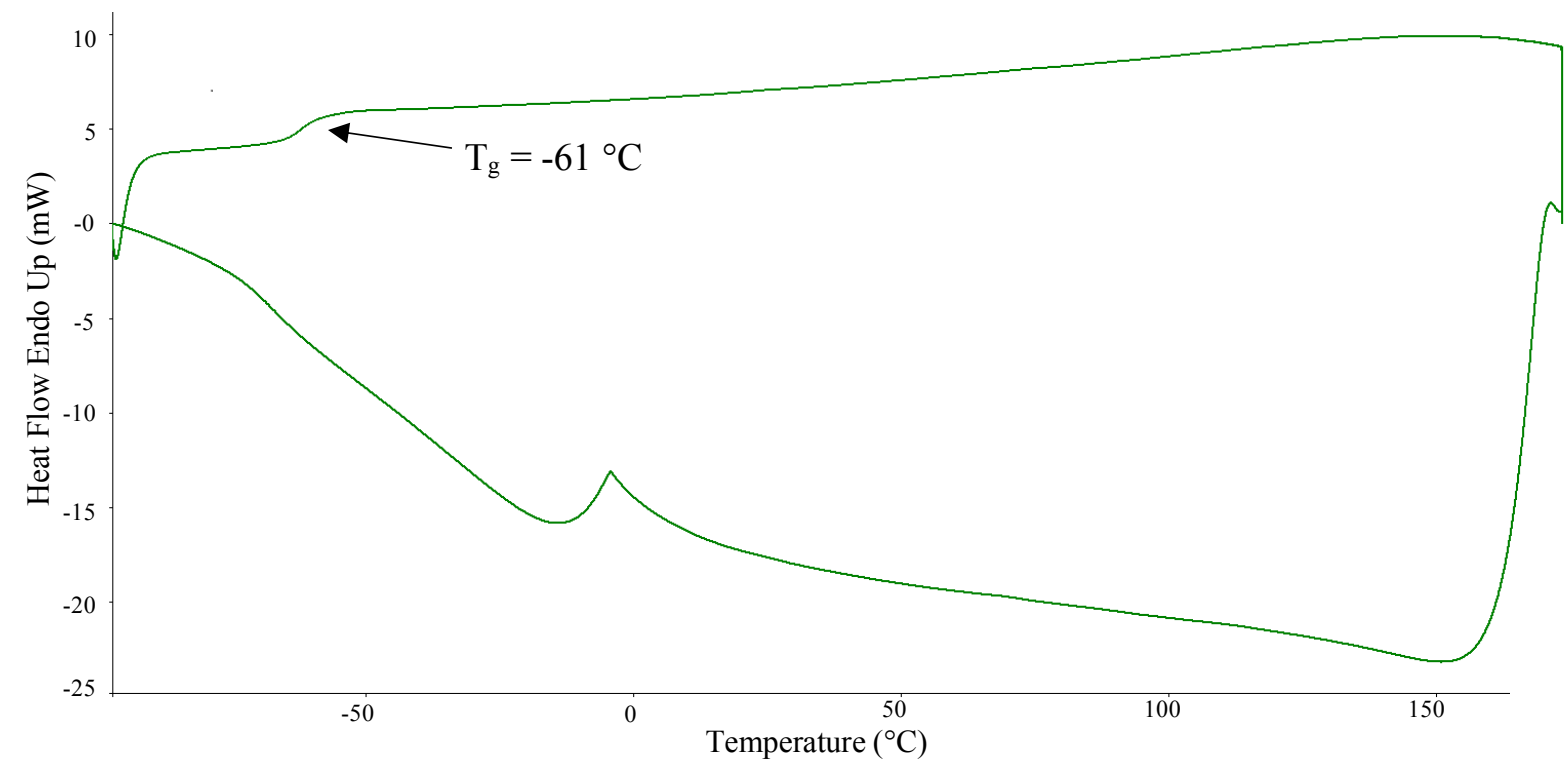

Figure S45. DSC curve of polyisoprene (entry 4). 


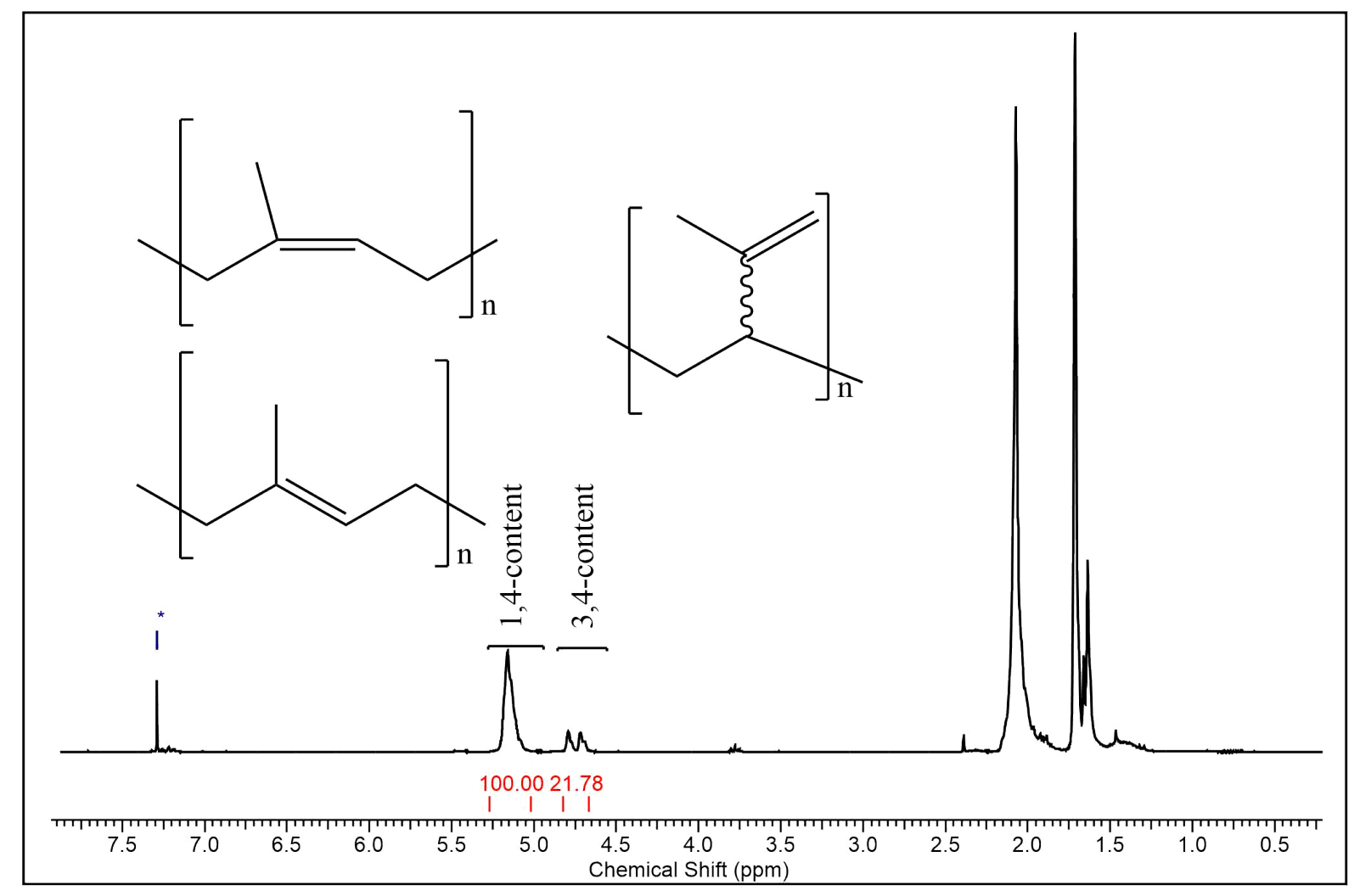

Figure S46. ${ }^{1} \mathrm{H}$ NMR spectrum (400 MHz) of polyisoprene (entry 14) in $\mathrm{CDCl}_{3}$ at $26{ }^{\circ} \mathrm{C}$.

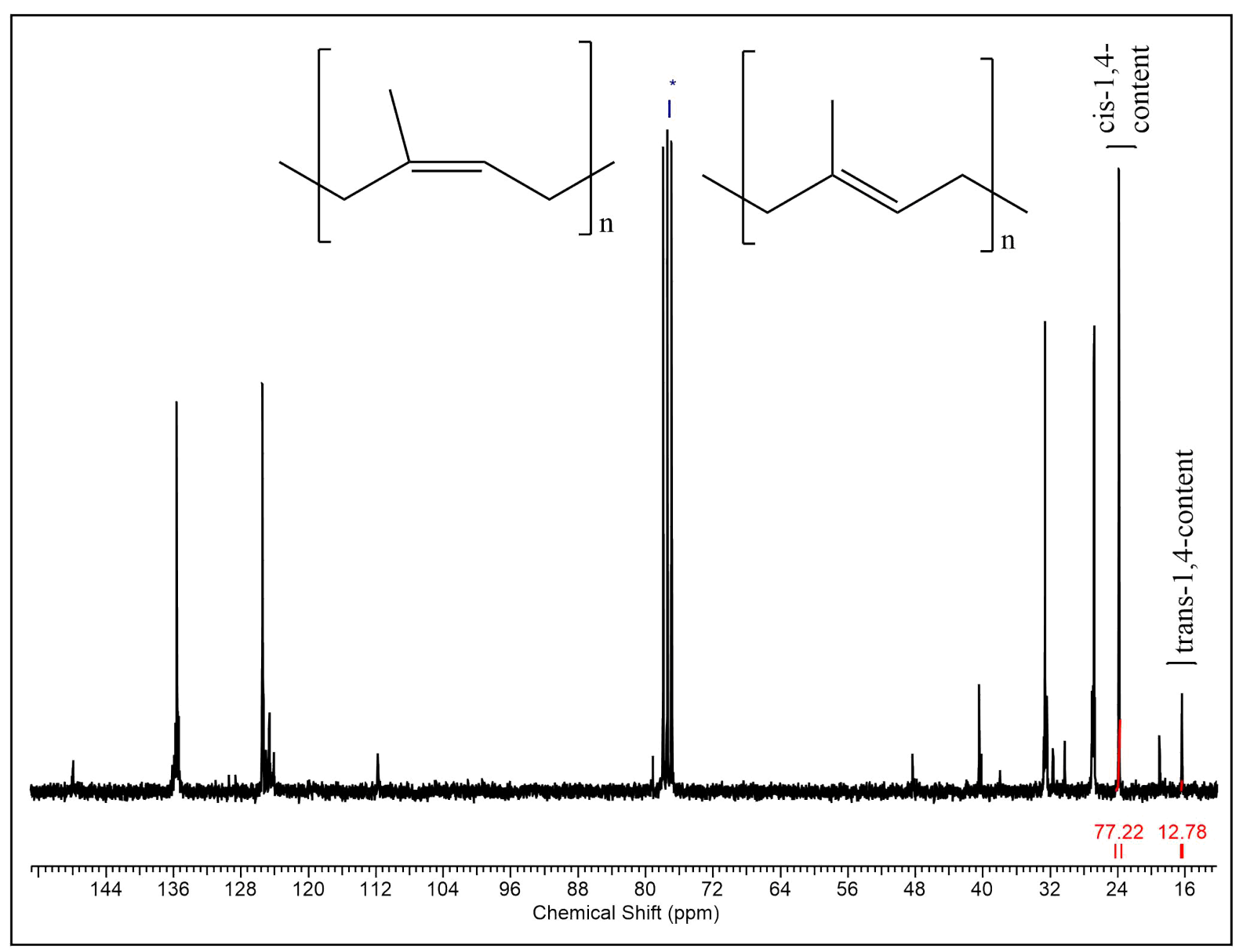

Figure S47. ${ }^{13} \mathrm{C}\left\{{ }^{1} \mathrm{H}\right\}$ NMR spectrum (101 MHz) of polyisoprene (entry 14) in $\mathrm{CDCl}_{3}$ at $26{ }^{\circ} \mathrm{C}$. 


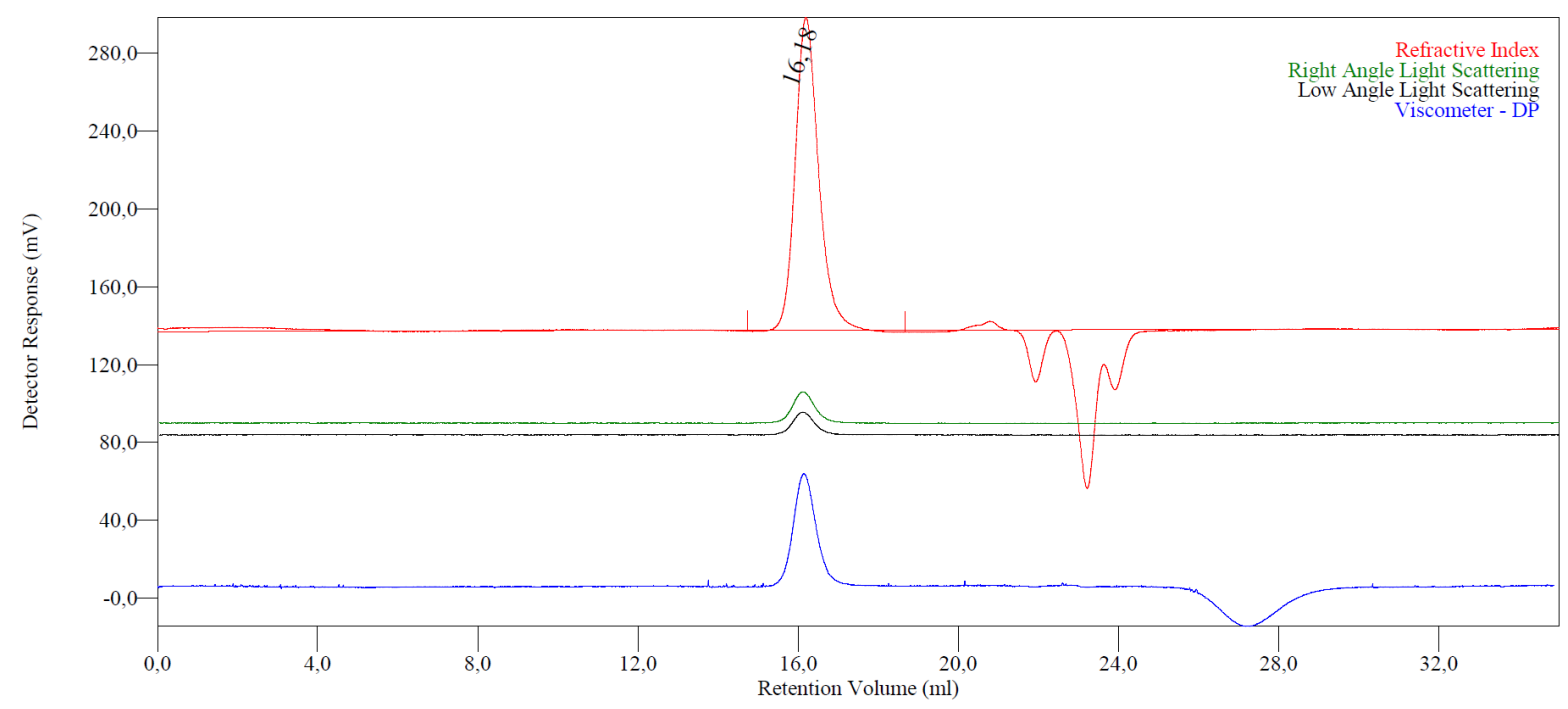

Figure S48. GPC curve of polyisoprene (entry 14).

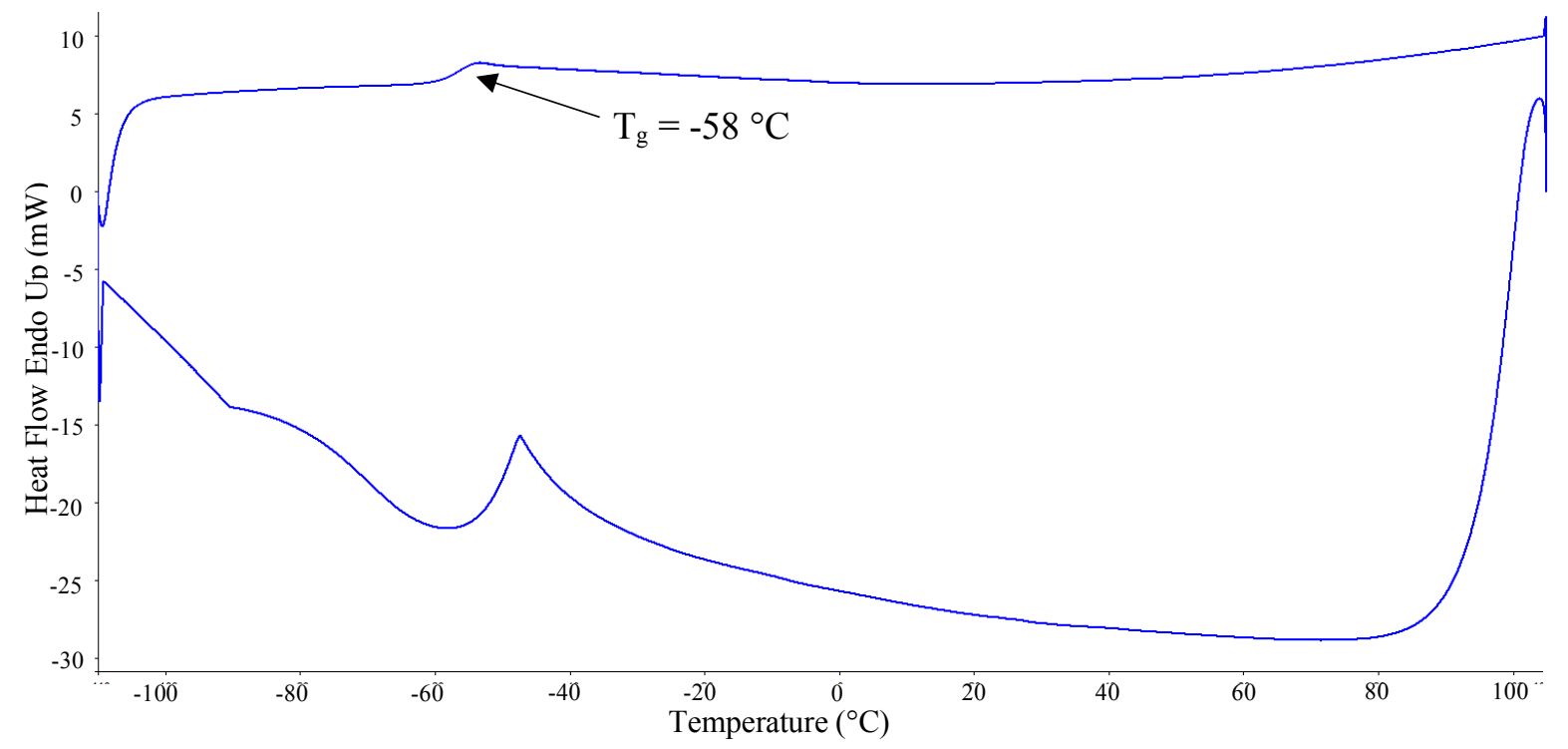

Figure S49. DSC curve of polyisoprene (entry 14). 